\title{
A search for diffuse bands in the circumstellar envelopes of post-AGB stars ${ }^{\star}, \star \star$
}

\author{
R. Luna ${ }^{1}$, N. L. J. Cox ${ }^{2}$, M. A. Satorre ${ }^{1}$, D. A. García Hernández ${ }^{3}$, O. Suárez ${ }^{4}$, and P. García Lario ${ }^{2}$ \\ ${ }^{1}$ Laboratorio de Astrofísica Experimental, Escuela Politécnica Superior de Alcoy, Universidad Politécnica de Valencia, \\ Plaza de Ferrándiz y Carbonell, 03801 Alcoy, Alicante, Spain \\ e-mail: ralunam@fis.upv.es \\ ${ }^{2}$ Herschel Science Centre, Research and Scientific Support Department of ESA, European Space Astronomy Centre, PO Box 78, \\ 28691 Villanueva de la Cañada, Madrid, Spain \\ 3 W. J. McDonald Observatory. The University of Texas at Austin. 1 University Station, C1400. Austin, TX 78712-0259, USA \\ ${ }^{4}$ LUAN, Université de Nice Sophia Antipolis, Parc Valrose, 06108 Nice Cedex 2, France
}

Received 27 March 2006 / Accepted 6 November 2007

\section{ABSTRACT}

\begin{abstract}
In this work we present the results of a systematic search for diffuse bands (DBs, hereafter) in the circumstellar envelopes of a carefully selected sample of post-AGB stars. We concentrated on analyzing 9 of the DBs most commonly found in the interstellar medium. The strength of these features is determined using high-resolution optical spectroscopy, and the results obtained are compared with literature data on field stars affected only by interstellar reddening. Based on the weak features observed in the subsample of postAGB stars dominated by circumstellar reddening, we conclude that the carrier(s) of these DBs must not be present in the circumstellar environment of these sources, or at least not under the excitation conditions in which DBs are formed. This conclusion is applicable to all the post-AGB stars studied, irrespective of the dominant chemistry or the spectral type of the star considered. A detailed radial velocity analysis of the features observed in individual sources confirms this result, as the Doppler shifts measured are found to be consistent with an interstellar origin.
\end{abstract}

Key words. stars: AGB and post-AGB - ISM: dust, extinction

\section{Introduction}

The diffuse interstellar bands (DIBs) are absorption features, showing a broad range of widths and strengths, which appear over-imposed on the spectra of bright stars whose lines of sight probe (extra)galactic diffuse-to-dense interstellar clouds. Currently, more than 200 DIBs have been identified and catalogued in the spectral range from 3600 to $10200 \AA$ (Jenniskens \& Désert 1994; Cox et al. 2005), the most studied ones being those found at 4430, 5780, 5797, and $6284 \AA$. Since their discovery (Heger 1922), they have been associated to the interstellar medium (ISM), because their strengths show a positive relationship with the observed extinction (Merrill 1936), as well as to the neutral sodium column density (Herbig 1993). Many carriers have been proposed, however, no unambiguous identification has yet been made and it is debated whether they arise from the dust or the gas components of the ISM (see reviews by Herbig 1995 and Sarre 2006). There is increasing observational evidence that the DIB carriers constitute a set of carbonaceous gas-phase molecules as shown from substructures resembling rotational contours in some bands (Sarre et al. 1995; Ehrenfreund \& Foing 1996). In particular, photo-UV-resistant organic molecules, such as carbon chains (Douglas 1977), PAHs (Salama et al. 1999; Allamandola et al. 1999), fullerenes

\footnotetext{
* Based on observations collected at the European Southern Observatory (Chile) and at the Spanish Observatorio del Roque de los Muchachos of the Instituto de Astrofísica de Canarias.

$\star \star$ Tables 5-8 are only available in electronic form at

http://www . aanda.org
}

(Foing \& Ehrenfreund 1994; Iglesias-Groth 2007), and / or buckyonions (Iglesias-Groth 2004), are promising candidates. The local interstellar environmental conditions set the balance of local formation and destruction of the carriers, as well as their level of ionisation and hydrogenation. The interstellar radiation field is one of the most important factors in this (Ruiterkamp et al. 2005).

There is a possible link between the DIB carriers and the carriers of the unidentified (aromatic) infrared bands (UIBs), the so-called PAH-DIB hypothesis (Crawford et al. 1985; Leger \& D’Hendecourt 1985; Van der Zwet \& Allamandola 1985). Thus, although PAHs are thought to reside and to be processed (ionisation, dehydrogenation, destruction) in the diffuse ISM, this does not exclude the scenario that these molecules (or their parent species) are produced elsewhere. Since circumstellar shells are sources of replenishment of the ISM, it has been argued that DIBs (and/or parent structures) may have a circumstellar origin, either in dense stellar winds or circumstellar shells, thus somehow contravening the name they were initially given. The suspected connection between DIB carriers and some carbon-rich compounds can be investigated attending to the usually known chemistry and physical properties of these circumstellar shells.

Observationally, the detection of diffuse bands (DBs, hereafter) around evolved stars is hampered by the fact that most of them are losing mass and are usually strongly variable and surrounded by very cool extended atmospheres where molecules are the dominant source of opacity. These stars are very difficult to model, and DBs are hardly detected (in absorption) against the forest of features attributed to molecular transitions that appear superimposed on the stellar continuum. This has 
hampered any systematic search for DBs in evolved stars in the past. Furthermore, if detected, it is difficult to determine whether the DBs are originating from the interstellar or the circumstellar environment, or even both.

In the face of these difficulties, Snow \& Wallerstein (1972) and Snow (1973) searched for circumstellar diffuse bands (at 4430, 5780, 5797, and $6614 \AA$ ) in 26 stars with suspected circumstellar dust shells / envelopes but found no evidence of their presence. Several other authors have since searched for and commented on the presence of diffuse bands and their interstellar or circumstellar nature, in spectra observed towards planetary nebulae (NGC 6210, NGC 7027, IC 351, and AFGL 2688 by Prichet \& Grillmair 1984; IRAS $21282+5050$ by Cohen \& Jones 1987; NGC 7027 and IRAS 21282+5050 by Le Bertre \& Lequeux 1992), a post-AGB star (HR 4049; Waters et al. 1989), and a carbon star (IRAS 07270-1921 or CGCS 1732; Le Bertre 1990). Le Bertre \& Lequeux (1993) studied a new sample consisting of carbon-, oxygen-, or nitrogen-rich mass-losing sources, such as (pre)planetary nebulae (BD+30 3639, CPD-56 8032, Hen 104), a carbon-rich RV Tauri star (AC Her), Wolf-Rayet stars (WR137, WR140), and post-AGB stars (HR 4049, HD 213985), revisiting several sources studied in the past (e.g. CS 776, NCG 7027, HR 4049, IRAS $21282+5050$ ). These authors did not find any evidence of circumstellar DBs, which refuted previous claims, and thus concluded that DBs are depleted in circumstellar environments. Notably, these authors did not detect any bands in the spectra of sources showing strong PAH emission (UIB) at mid-infrared wavelengths. This suggested that carrier molecules, if present, in circumstellar envelopes are in a different state of ionisation / hydrogenation than in the ISM. Strong DBs were detected toward carbon-rich sources that do not show PAH emission, as well as toward most of their oxygen-rich and nitrogen-rich sources in the sample, although in all cases the observed DBs could be attributed to the interstellar material in the lines of sight. For unexplained reasons, enhanced DBs were detected toward WN stars and LBVs. Exceptionally, narrow emission features possibly also related to DIBs have been observed toward the Red Rectangle (Scarrott et al. 1992), although their identification and nature remain controversial.

A largely unexplored alternative exists. This concerns the socalled post-AGB stars that are in a short-lived transition phase between the asymptotic giant branch (AGB) and the planetary nebula (PN) stages, evolving very rapidly in the H-R diagram while they are still surrounded by the remnant of the AGB circumstellar shell. Post-AGB stars show all possible spectral types from $\mathrm{M}$ to $\mathrm{B}$ in what probably represents an evolutionary sequence of increasingly effective temperature on their way to becoming PNe (García-Lario et al. 1997b). This means that in these stars we should be able to easily detect DBs formed in the remnant AGB shell superimposed on the intermediate or early-type spectrum of the central star without the confusion originating from the presence of molecular bands in AGB stars. Interestingly, while many of these DBs are common to those observed in the ISM, the relative ratios are sometimes found to be very different (García-Lario et al. 1999). These circumstellar DBs could form and survive for some time under conditions that might be substantially different from those found in the ISM in terms of density, UV radiation field, etc. and could hold the key to understanding and solving this longstanding problem. Another advantage is that the chemical composition of the gas and dust in these shells can be determined easily from observations in the optical, infrared, $\mathrm{mm} / \mathrm{sub}-\mathrm{mm}$, or radio wavelengths. In addition, post-AGB stars are located in many cases at relative high galactic latitudes and are as such only affected by low interstellar reddening. This facilitates the attribution of a circumstellar origin to the features observed.

The potential formation of DBs around post-AGB stars has, however, so far only been explored occasionally and for a limited number of sources. Nevertheless, the presence of strong DBs has been reported in the optical spectra of a few post-AGB stars (Le Bertre \& Lequeux 1993; García-Lario et al. 1999; Zacs et al. 1999a, 2001; Klochkova et al. 1999, 2000; Kendall et al. 2002) and some carbon-rich (barium) stars (Zacs et al. 2003). In several cases, tentative claims have been put forward for DBs detected at radial velocities coinciding with the photospheric absorption lines or shell/envelope expansion velocity (IRAS 04296+3429 by Klochkova et al. 1999; and HD 179821 by Zacs et al. 1999a). All other studies listed above gave non-conclusive results.

Another method recently employed utilised nearby background stars to probe the circumstellar environments of the carbon star IRC+10216 (Kendall et al. 2002) and the helix PN (Mauron \& Kendall 2004). Again, no circumstellar DBs were detected, confirming the lack of DIB carriers in these environments.

In this work we present the first systematic survey to detect the circumstellar DBs (DCBs) in a carefully selected sample of galactic post-AGB stars ${ }^{1}$. The goal is to perform a detailed analysis of the differential properties observed in the DBs associated to post-AGB stars in comparison with the standard DBs observed towards reddened, early-type field stars (where these bands are expected to be essentially of interstellar origin).

To perform this task we studied the intensity of 9 of the strongest absorption features identified as DBs in the spectral range 4000-10000 $\AA$ using high-resolution optical spectroscopy. The comparison of the properties observed in carbon-rich and oxygen-rich post-AGB stars is used to test the carbon-rich nature of the DB carrier(s) and determine which of the detected DBs are most probably of circumstellar origin (if any).

In Sect. 2 we describe the observations made and the data reduction process. The strategy followed in our analysis is presented in Sect. 3. The main results are discussed in Sect. 4 as a function of various observational parameters. Finally, the conclusions are presented in Sect. 5.

\section{Observations and data reduction}

The high-resolution Echelle spectroscopic data analysed in this paper were taken with a wide variety of instruments and telescopes over the period 1993-2003. Originally, these observations were carried out for chemical abundance analysis purposes, and they correspond to observations performed using the Utrecht Echelle Spectrograph (UES) at the William Herschel Telescope (WHT $4.2 \mathrm{~m}$ ) and the High Resolution Spectrograph (SARG) at the Telescopio Nazionale Galileo (TNG $3.58 \mathrm{~m}$ ), both in the Spanish Observatorio del Roque de los Muchachos (La Palma, Spain); the UV-Visual Echelle Spectrograph (UVES) installed at the Very Large Telescope-U2 (VLT $8 \mathrm{~m}$ ) in Paranal Observatory (Chile); and the ESO Multi-Mode Instrument (EMMI) at the New Technology Telescope (NTT $3.5 \mathrm{~m}$ ) and the Fiber-fed Extended Range Optical Spectrograph (FEROS) at the ESO $1.52 \mathrm{~m}$ telescope in La Silla Observatory (Chile).

The spectra obtained cover a wide wavelength range (usually from 4000 to $10000 \AA$ ) with a resolving power in the

\footnotetext{
1 The sample also includes three very young planetary nebulae, which are here also considered post-AGB stars in a broad sense.
} 


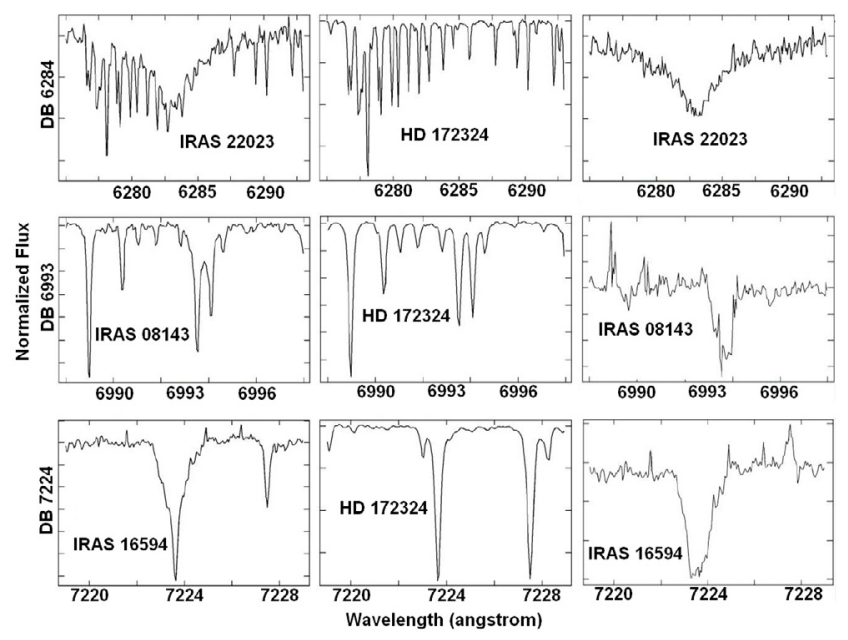

Fig. 1. Removal of telluric lines at 6284, 6993, and $7224 \AA$ A. A few examples are shown before (left) and after correction (right). In the middle panel we show the stellar spectrum of HD 172324 (B9Ib), one of the sample stars, which was used as telluric divisor (see text).

range 50000-100000 at $5500 \AA$, depending on the instrument set-up. The exposure times are variable, depending on the brightness of the source, but typically $\sim 30 \mathrm{~min}$, leading to a signal-tonoise of 20-200 over the spectral range considered.

The two-dimensional spectra were reduced following the standard procedure for echelle spectroscopy using IRAF astronomical routines. The process includes identification of bad pixels, bias determination and scattered light subtraction, flat-field correction, order extraction, and wavelength calibration. For the DBs at 6284,6993 , and $7224 \AA$, strongly affected by terrestrial features, the spectrum of HD 172324 (B9Ib), one of the sample stars very little affected by extinction, was used to remove the telluric absorption lines ${ }^{2}$ (see Fig. 1).

\section{Selection of the sample}

For our systematic study of the presence of DCBs in post-AGB stars, we carefully selected a sample of 33 sources from the literature trying to cover as much as possible of a wide variety of observational properties such as the chemistry of the circumstellar envelope (carbon-rich and oxygen-rich) or the spectral type of the central stars ${ }^{3}$. Priority was given to sources located at high galactic latitudes showing a strong colour excess $E(B-V)$ (as these sources are most likely dominated by circumstellar extinction) and to those for which a high radial velocity has been reported in the literature (since this may later facilitate the identification of spectral features of circumstellar origin).

The main objective is to understand whether systematic differences are detected which depend on one (or more) of the above observational parameters. The list of stars selected for analysis, most of them IRAS sources belonging to the GLMP catalogue of post-AGB stars (García-Lario et al. 1997a;

\footnotetext{
2 Note that the initial strategy was to use the spectrum of a hot, rapidly rotating star observed on the same night for this purpose, but it was found that the latter showed faint but detectable DBs in its spectrum, which did not allow us to perform this correction properly. In contrast, the 6284,6993 , and $7224 \AA$ DBs were found to be totally absent in HD 172324.

3 Stars with spectral types later than G- were discarded for the analysis, as their continuum is dominated by the molecular bands, which makes identifying DBs a very difficult task.
}

Suárez 2004), is displayed in Table 1, where a summary of the observations is presented. In Table 2, additional information is given on the sources included in our observing programme. This includes the colour excess $E(B-V)$, dominant chemistry (carbon-rich or oxygen-rich), spectral type, and galactic coordinates (GLON, GLAT), as well as the bibliographic references from which this information was extracted.

The spectral regions corresponding to 9 different DBs among the strongest ones reported in the literature were investigated in detail for each of the sources included in our sample. Table 3 lists the accurate wavelengths $\left(\lambda_{0}\right)$ corresponding to each of these features, taken from Galazutdinov et al. (2000), as well as their central depth $A_{\mathrm{C}}$ and sensitivity to the extinction, measured as $E W / E(B-V)$, observed toward the star HD 183143 (B7 I; $E(B-V)=1.28 \mathrm{mag}$ ), which is usually taken as the prototype star in the analysis of DIBs (Herbig 1995). All the DBs selected for analysis are within the optical domain, and they are referred to in the literature as the $5780,5797,5850,6196,6284$, $6379,6614,6993$, and $7224 \AA$ features. Other well-known DBs at 4430 and $6177 \AA$ are even stronger than the selected ones, but they have been discarded for study because of the difficulty detecting their extremely broad (and relatively shallow) profiles $\left(F W H M>17 \AA ; A_{\mathrm{c}}<0.1\right)$ in our high-resolution spectra.

\section{Discussion}

\subsection{DB strength vs. extinction in field stars}

Although it is generally accepted that there is a tight correlation between the equivalent width of DBs and the value of $E(B-V)$ in field stars dominated by interstellar reddening, the available results in the literature generally cover only the stronger DBs ( $\lambda \lambda$ 4430, 5780, 5797, and 6284). In addition, they are usually based on outdated data obtained with poor spectroscopic resolution (sometimes, even below $R=1000$ ). Unfortunately, studies covering other DBs and/or based on high-resolution spectroscopy are scarce. Prior to deriving any conclusion about the existence (or not) of a similar correlation between DB strength and extinction in our sample of post-AGB stars, it is necessary to establish this dependency for each of the 9 DBs observed toward field stars.

For this purpose, we again derived these correlation parameters ourselves using a large number of early-type stars taken from Weselak et al. (2001), Thorburn et al. (2003), and Megier et al. (2005), for which accurate DB strength measurements are available covering a wide range of extinction values. The Weselak et al. sample contains 41 stars observed at $R \sim 64000$, for which equivalent width data are available corresponding to the DB centred at $6614 \AA$. The Thorburn et al. sample comprises 53 stars observed with spectral resolution $R \sim 38000$, which were originally used to study the DBs centred at 5780, 5797, 6196, 6284, 6379, and $6614 \AA$. Finally, the Megier et al. sample comprises 49 stars observed with spectral resolution $R \sim 64000$, which were originally used to study the DBs centred at 5780, 5797, 5850, 6196, 6284, 6379, and $6614 \AA$ A. Four additional stars taken from Jenniskens \& Désert (1994), observed at $R \sim 20000$, were also used to derive the correlation parameters associated to the DBs centred at 6993 and $7224 \AA$, for which measurements are much scarcer in the literature. The sources included in these four samples are expected to follow behaviour representative of field stars affected only by interstellar extinction. Figure 2 shows all the equivalent width measurements used in our analysis, plotted as a function of the interstellar extinction, measured as $E(B-V)$. 
Table 1. Observation log.

\begin{tabular}{|c|c|c|c|c|c|c|c|}
\hline Name & Other names & Observing date & Observatory & Telescope & Instrument & Spectral range $(\AA)$ & Resolution \\
\hline IRAS $01005+7910$ & & 12.09 .03 & La Palma & TNG $(3.58 \mathrm{~m})$ & SARG & $4960-10110$ & 50000 \\
\hline IRAS Z02229+6208 & & 12.09 .03 & La Palma & TNG (3.58 m) & SARG & $4960-10110$ & 50000 \\
\hline IRAS 04296+3429 & & 22.08 .94 & La Palma & WHT (4.2 m) & UES & $5570-10200$ & 55000 \\
\hline IRAS $05113+1347$ & & 23.02 .94 & La Palma & WHT (4.2 m) & UES & $4480-10200$ & 55000 \\
\hline IRAS 05251-1244 & IC 418 & 12.09 .03 & La Palma & TNG $(3.58 \mathrm{~m})$ & SARG & $4960-10110$ & 50000 \\
\hline IRAS $05341+0852$ & & 30.09 .98 & ESO-La Silla & NTT (3.58 m) & EMMI & $5570-10040$ & 65000 \\
\hline IRAS 06530-0213 & & 12.01 .01 & ESO-Paranal & VLT-U2 (8 m) & UVES & $4790-6810$ & 100000 \\
\hline IRAS $07134+1005$ & HD 56126 & 20.12 .93 & La Palma & WHT (4.2 m) & UES & $3980-10400$ & 55000 \\
\hline IRAS 08005-2356 & & 02.03 .94 & La Palma & WHT (4.2 m) & UES & $3690-11050$ & 55000 \\
\hline IRAS 08143-4406 & & 16.01 .01 & ESO-Paranal & VLT-U2 (8 m) & UVES & $3750-10520$ & 100000 \\
\hline IRAS 08544-4431 & & 26.01 .99 & ESO-La Silla & ESO-1.52 & FEROS & $3700-8860$ & 50000 \\
\hline IRAS $12175-5338$ & SAO 239853 & 24.03 .00 & ESO-La Silla & ESO-1.52 & FEROS & $3730-8850$ & 50000 \\
\hline IRAS 16594-4656 & & 20.05 .00 & ESO-Paranal & VLT-U2 (8 m) & UVES & $3750-10520$ & 100000 \\
\hline IRAS 17086-2403 & & 14.07.01 & La Palma & WHT (4.2 m) & UES & $4300-9000$ & 50000 \\
\hline IRAS 17097-3210 & HD 155448 & 07.07.97 & ESO-La Silla & NTT (3.58 m) & EMMI & $5800-10430$ & 60000 \\
\hline IRAS 17150-3224 & RAFGL 6815 & 10.06 .00 & ESO-Paranal & VLT-U2 (8 m) & UVES & $3750-10520$ & 100000 \\
\hline IRAS 17245-3951 & & 21.05 .00 & ESO-Paranal & VLT-U2 (8 m) & UVES & $3750-10520$ & 100000 \\
\hline IRAS 17395-0841 & & 13.07.01 & La Palma & WHT (4.2 m) & UES & $4300-9000$ & 55000 \\
\hline IRAS 17423-1755 & Hen 3-1475 & 13.07.01 & La Palma & WHT (4.2 m) & UES & $4300-9000$ & 55000 \\
\hline IRAS $17436+5003$ & HD 161796 & 01.09 .94 & La Palma & WHT (4.2 m) & UES & $3650-10200$ & 55000 \\
\hline IRAS 18025-3906 & & 30.09 .98 & ESO-La Silla & NTT (3.58 m) & EMMI & $3980-10430$ & 65000 \\
\hline \multirow[t]{2}{*}{ IRAS $18062+2410$} & HD 341617 & 13.07 .01 & La Palma & WHT (4.2 m) & UES & $4300-9000$ & 50000 \\
\hline & HD 172324 & 08.08 .95 & ESO-La Silla & NTT (3.58 m) & EMMI & $3650-10240$ & 55000 \\
\hline IRAS 19114+0002 & HD 179821 & 07.07 .97 & ESO-La Silla & NTT (3.58 m) & EMMI & $5800-10430$ & 60000 \\
\hline IRAS $19200+3457$ & LS II +34 1 & 11.09 .03 & La Palma & TNG (3.58 m) & SARG & $4960-10110$ & 50000 \\
\hline IRAS $19386+0155$ & & 30.09 .98 & ESO-La Silla & NTT (3.58 m) & EMMI & $5980-8320$ & 65000 \\
\hline IRAS 19500-1709 & HD 187885 & 07.08 .95 & ESO-La Silla & NTT (3.58 m) & EMMI & $3650-10040$ & 55000 \\
\hline IRAS $20000+3239$ & & 23.08 .94 & La Palma & WHT (4.2 m) & UES & $4440-10040$ & 50000 \\
\hline IRAS $20462+3416$ & LS II +34 26 & 17.08 .96 & La Palma & WHT (4.2 m) & UES & $5300-9380$ & 55000 \\
\hline IRAS $22023+5249$ & LS III +52 24 & 14.07 .01 & La Palma & WHT (4.2 m) & UES & $4300-9000$ & 55000 \\
\hline IRAS $22223+4327$ & $\mathrm{BD}+424388$ & 24.08 .94 & La Palma & WHT (4.2 m) & UES & $4440-10040$ & 50000 \\
\hline IRAS $22272+5435$ & HD 235858 & 23.08 .94 & La Palma & WHT (4.2 m) & UES & $4440-10220$ & 55000 \\
\hline IRAS 23304+6147 & & 23.08 .94 & La Palma & WHT (4.2 m) & UES & $4440-10040$ & 55000 \\
\hline
\end{tabular}

For each DB under analysis we applied a linear fit to the data available. We also imposed the condition $E W=0$ for $E(B-V)=0$, i.e.: $E W=a \cdot E(B-V)$, where $a$ is a constant that represents the equivalent width per extinction unity. In practice this is equivalent to assuming that there is a direct link between the DB carrier(s) and the material responsible for the extinction observed in the ISM. These fits represent the DB strength expected as a function of the colour excess for any given source in which interstellar reddening is the dominant contributor to the overall extinction. These are represented by solid lines in Fig. 2. The slopes $(E W / E(B-V))$ and correlation coefficients $r$ of the linear fits are given in Table 3.

As we can see, a reasonable correlation between equivalent width and $E(B-V)$ is always found, although in some cases the dispersion is considerable. The new results obtained agree, as do those derived for the prototype star HD 183143 by Herbig (1995), although the 5780 and 6284 DIBs are significantly stronger toward the latter, probably due to local environmental conditions. These results give confidence when proceeding with the study of the post-AGB stars in our sample, based on the assumption that the above values can be taken as references for the subsequent analysis.

\subsection{DB strength vs. extinction in post-AGB stars}

In Table 5 (online only) we show the equivalent width of each of the 9 DBs considered in our analysis for every post-AGB star in the sample, as determined from the available high-resolution spectra. Note that three of these bands are strongly affected by telluric contamination, namely those centred at 6284,6993 , and $7224 \AA$. For these features, the telluric component was carefully removed before determining their $E W$ (see Figs. 1 and 3; Sect. 2). The other DBs were measured directly on the normalised spectra. We note here that in none of the spectra did we find evidence of DB features in emission.

The resulting values are plotted in Fig. 4 as a function of the colour excess $E(B-V)$. The values of $E(B-V)$ used to produce this figure are taken directly from the literature (see Table 2) or estimated from the available information on spectral type and photometry by comparing the observed $B$ and $V$ magnitudes with the intrinsic $B-V$ colours expected for stars of the same spectral type and luminosity class $\mathrm{I}^{4}$.

Figure 4 shows the overall results for the 9 DBs studied. In general we find that the equivalent width of the observed features seems to still be correlated with the value of $E(B-V)$. However, in contrast to the results obtained for the field stars (shown in Fig. 2), this correlation is now very weak in some cases, and we identify a much larger number of outliers.

Usually, for a given extinction $E(B-V)$, the measured equivalent widths in post-AGB stars are well below the expected

\footnotetext{
4 A luminosity class I is adopted because this is the class corresponding to low-gravity stars. Note that post-AGB stars only look like supergiants, but they are not genuine population I massive super-giant stars (Fitzgerald 1970). Values quoted by different authors are generally in good agreement, and the discrepancies found, when relevant, are reflected in the associated errors provided in Table 2.
} 
Table 2. Main characteristics of the post-AGB stars selected for the analysis.

\begin{tabular}{|c|c|c|c|c|c|c|c|c|}
\hline IRAS name & $\bar{E} E(B-V)$ & $\overline{\overline{\text { Ref. }}}$ & Chemistry & Ref. & Sp.type & Ref. & GLON. & GLAT. \\
\hline $01005+7910$ & $0.2 \pm 0.1$ & $(25)$ & $\mathrm{C}$ & $(25)$ & B2 & $(25)$ & 123.57 & +16.59 \\
\hline $02229+6208$ & $1.67 \pm 0.09$ & (17) & $\mathrm{C}$ & (37) & G9Ia & (37) & 133.73 & +1.50 \\
\hline $04296+3429$ & $1.3 \pm 0.1$ & $(7,15,36)$ & $\mathrm{C}$ & (27) & G0Ia & (3) & 166.24 & -9.05 \\
\hline $05113+1347$ & $1.1 \pm 0.2$ & $(26,36,40)$ & $\mathrm{C}$ & (26) & G5I & (40) & 188.86 & -14.29 \\
\hline 05251-1244 & $0.23 \pm 0.09$ & (35) & $\mathrm{C}$ & (42) & $\mathrm{PN} / 07 \mathrm{f}$ & (14) & 215.21 & -24.28 \\
\hline $05341+0852$ & $1.65 \pm 0.09$ & $(7,17,40)$ & $\mathrm{C}$ & (26) & F5I & (15) & 196.19 & -12.14 \\
\hline $06530-0213$ & $1.7 \pm 0.2$ & $(36,40)$ & $\mathrm{C}$ & (18) & F5I & (18) & 215.44 & -0.13 \\
\hline $07134+1005$ & $0.4 \pm 0.1$ & $(15,40)$ & $\mathrm{C}$ & (26) & F7 Ie & (40) & 206.75 & +9.99 \\
\hline 08005-2356 & $0.7 \pm 0.3$ & $(15,40)$ & $\mathrm{O}$ & (5) & F5I & (3) & 242.36 & +3.58 \\
\hline 08143-4406 & $0.8 \pm 0.1$ & $(36,40)$ & $\mathrm{C}$ & (39) & F8I & (36) & 260.83 & -5.07 \\
\hline 08544-4431 & $1.45 \pm 0.09$ & (29) & $\mathrm{O}$ & (29) & F3 & (29) & 265.50 & +0.39 \\
\hline $12175-5338$ & $0.25 \pm 0.09$ & $(19,38$ & $\mathrm{C}$ & (46) & A9Iab & (32) & 298.30 & +8.67 \\
\hline $16594-4656$ & $2.2 \pm 0.3$ & $(20,40,44)$ & $\mathrm{C}$ & (9) & B7 & (44) & 340.39 & -3.29 \\
\hline $17086-2403$ & $0.86 \pm 0.09$ & (40) & C? & (10) & $\mathrm{PN}+\mathrm{G} 5 ?$ & (34) & 359.84 & +8.99 \\
\hline $17097-3210$ & $0.06 \pm 0.05$ & (30) & C & (45) & B9 & (30) & 353.36 & +4.03 \\
\hline $17150-3224$ & $0.68 \pm 0.09$ & (23) & $\mathrm{O}$ & (23) & G2I & (23) & 353.84 & +2.98 \\
\hline $17245-3951$ & $1.0 \pm 0.1$ & (40) & $\mathrm{O}$ & (20) & F6I & (40) & 348.81 & -2.84 \\
\hline $17395-0841$ & $1.1 \pm 0.2$ & $(11,40)$ & $\mathrm{O}$ ? & (10) & $\mathrm{PN}+\mathrm{G}$ & (11) & 17.02 & +11.10 \\
\hline 17423-1755 & $1.13 \pm 0.09$ & $(11,40)$ & $\mathrm{O}$ & (41) & $\mathrm{Be}$ & (11) & 9.36 & +5.78 \\
\hline $17436+5003$ & $0.24 \pm 0.09$ & (19) & $\mathrm{O}$ & (4) & F3 Ib & (48) & 77.13 & +30.87 \\
\hline $18025-3906$ & $1.15 \pm 0.09$ & $(22,40)$ & $\mathrm{O}$ & (24) & G1I & (40) & 353.27 & -8.72 \\
\hline $18062+2410$ & $0.6 \pm 0.2$ & $(33,40)$ & $\mathrm{O}$ & (33) & B1 I & (33) & 50.67 & +19.79 \\
\hline HD 172324 & $0.03 \pm 0.02$ & (6) & $\mathrm{O}$ & (1) & B9Ib & (1) & 66.18 & +18.58 \\
\hline $19114+0002$ & $0.60 \pm 0.05$ & $(19,40)$ & $\mathrm{O}$ & (4) & G5 Ia & (3) & 35.62 & -4.96 \\
\hline $19200+3457$ & $0.3 \pm 0.1$ & (11) & $\mathrm{O}$ ? & (11) & B & (11) & 67.57 & +9.51 \\
\hline $19386+0155$ & $1.05 \pm 0.09$ & (2) & $\mathrm{O}$ & (28) & F5I & (31) & 40.51 & -10.09 \\
\hline $19500-1709$ & $0.37 \pm 0.09$ & (19) & $\mathrm{C}$ & (47) & F2-6 Ia & (3) & 23.98 & -21.04 \\
\hline $20000+3239$ & $1.6 \pm 0.1$ & $(15,26$ & $\mathrm{C}$ & (21) & G8Ia & (21) & 69.68 & +1.16 \\
\hline $20462+3416$ & $0.38 \pm 0.09$ & $(8,43)$ & $\mathrm{O}$ & (8) & B 1.5 & (8) & 76.60 & -5.75 \\
\hline $22023+5249$ & $0.52 \pm 0.09$ & (12) & $\mathrm{O}$ & (13) & B & (12) & 99.30 & -1.96 \\
\hline $22223+4327$ & $0.2 \pm 0.1$ & $(15,26)$ & $\mathrm{C}$ & (26) & G0 Ia & (15) & 96.75 & -11.56 \\
\hline $22272+5435$ & $0.9 \pm 0.2$ & (15) & $\mathrm{C}$ & (16) & G5 Ia & (15) & 103.35 & -2.52 \\
\hline $23304+6147$ & $1.4 \pm 0.2$ & $(15,36)$ & $\mathrm{C}$ & (15) & G2 Ia & (15) & 113.86 & +0.59 \\
\hline
\end{tabular}

(1) Arellano et al. (2001);

(2) Arkhipova et al. (2000);

(3) Bakker et al. (1997);

(4) Bujarrabal et al. (1992);

(5) Desmurs et al. (2002);

(6) Fernie (1983);

(7) Fujii et al. (2002);

(8) García-Lario et al. (1997a);

(9) García-Lario et al. (1999);

(10) García-Lario (priv. comm.);

(11) Gauba et al. (2003);

(12) Gauba \& Parthasarathy (2003);
(13) Gauba \& Parthasarathy (2004);

(14) Heap \& Augensen (1987);

(15) Hrivnak (1995);

(16) Hrivnak \& Kwok (1991);

(17) Hrivnak \& Kwok (1999);

(18) Hrivnak \& Reddy (2003);

(19) Hrivnak et al. (1989);

(20) Hrivnak et al. (1999);

(21) Hrivnak et al. (2000);

(22) Hu et al. (1993a);

(23) Hu et al. (1993b);

(24) Hu et al. (1994);
(25) Klochkova et al. (2002); (26) Kwok et al. (1995);

(27) Kwok et al. (1999);

(28) Lewis (2000);

(29) Maas et al. (2003);

(30) Malfait et al. (1998);

(31) Meixner et al. (1999);

(32) Oudmaijer et al. (1992);

(33) Parthasarathy et al. (2000a);

(34) Parthasarathy et al. (2000b);

(35) Pottasch et al. (2004);

(36) Reddy \& Parthasarathy (1996);
(37) Reddy et al. (1999);

(38) Reed \& Vance (1996);

(39) Reyniers et al. (2004);

(40) Suárez et al. (2006);

(41) te Lintel Hekkert et al. (1991);

(42) Torres-Peimbert et al. (1980);

(43) Turner \& Drilling (1984);

(44) van de Steene \& van Hoof (2003);

(45) van der Veen et al. (1989);

(46) van Winckel (1997);

(47) van Winckel \& Reyniers (2000);

(48) Volk \& Kwok (1989). values. Only a subset of sources follow exactly the same behaviour as observed in field stars. We interpret this result as the consequence of the absence (or at least the under-abundance) of the DB carriers in the circumstellar envelopes of most of these post-AGB stars, but further analysis is needed to confirm that there is no other alternative explanation.

\subsection{Interstellar vs. circumstellar extinction}

To determine whether our preliminary hypothesis is consistent with the measurements presented here, it is necessary to take into account that, in general, the overall extinction observed towards a given source in the sky is the result of the combined effect of the contribution from the ISM and of the internal extinction produced in the circumstellar shell. Making this distinction is generally not important in field stars, since for them the latter contribution is negligible. However, the situation is completely different for the evolved stars in our sample because, in many cases, the observed reddening almost exclusively has a circumstellar origin.

Disentangling interstellar versus circumstellar extinction for a given source is a very difficult task, if we need to rely only on the available observations. The only option we have is to use a statistical approach to estimate whether the observed extinction tends to corresponds to one or another component.

For this we represent in Fig. 5 the $E(B-V)$ versus galactic latitude distribution of the post-AGB stars in our sample and compare this distribution with what is shown by field stars taken from the catalogue of Guarinos (Guarinos 1988a,b, 1997). This catalogue, also used for the study of DB strengths, contains observations of 270 early-type field stars homogeneously distributed along the galactic plane (but excluding the galactic bulge), located at a variety of galactic latitudes, and their value of $E(B-V)$ has been previously determined.

Figure 5 shows clearly that a subsample of post-AGB stars are clear outliers in this plot. This indicates that the 


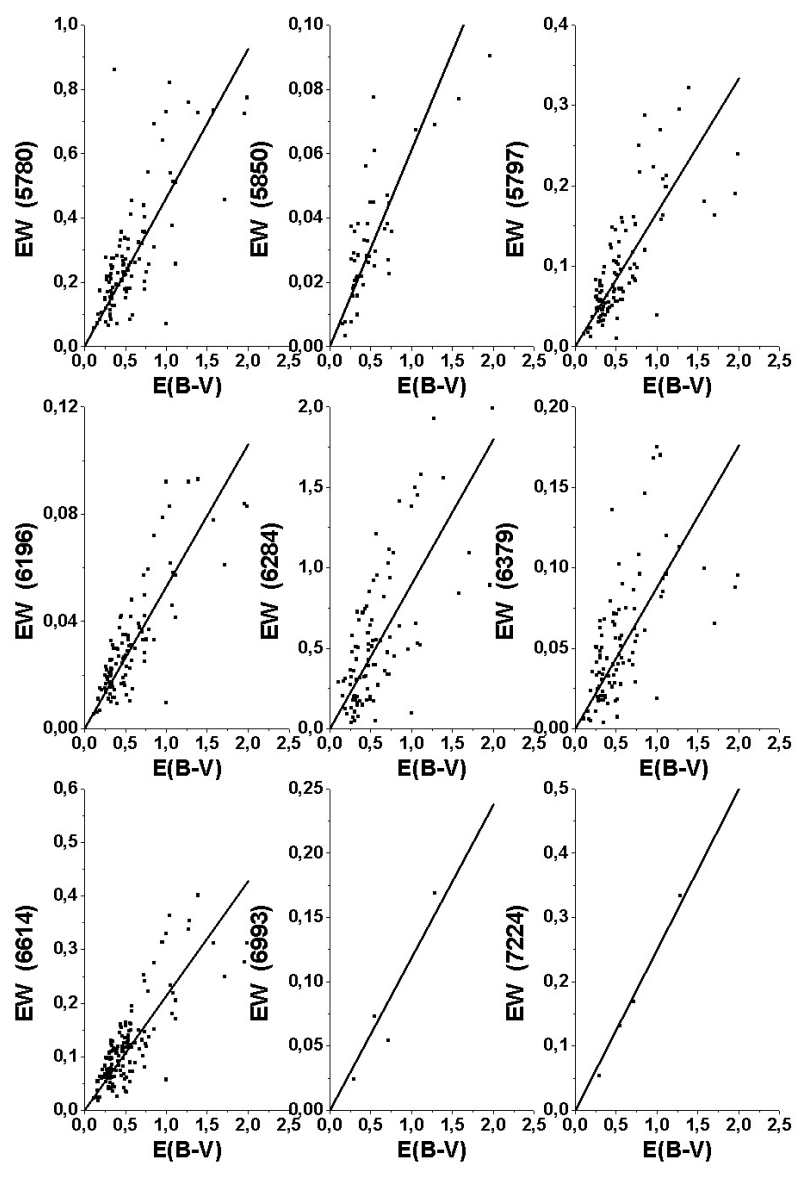

Fig. 2. Equivalent width measurements taken from the literature for field stars, plotted as a function of $E(B-V)$. Table 3 gives slopes $E W / E(B-V)$ and correlations $r$ for the linear fits.

Table 3. Main characteristics of the selected DBs (central depth $A_{\mathrm{C}}$ and normalised equivalent width $E W / E(B-V)$ ), as measured towards the prototype star HD 183143 (Herbig 1995).

\begin{tabular}{cccccc}
\hline \hline & & \multicolumn{3}{c}{ HD 183143 } & \multicolumn{2}{c}{ This work } \\
\cline { 3 - 6 } $\mathrm{DB}$ & $\lambda_{0}$ & $A_{\mathrm{c}}$ & $\begin{array}{c}E W / E(B-V) \\
(\AA / \mathrm{\AA ag})\end{array}$ & $\begin{array}{c}E W / E(B-V) \\
(\AA / \mathrm{mag})\end{array}$ & $r$ \\
\hline$\AA \AA ⿻$ & $(\AA)$ & & 0.63 & 0.46 & 0.74 \\
5780 & 5780.37 & 0.32 & 0.19 & 0.17 & 0.73 \\
5797 & 5796.96 & 0.20 & 0.06 & 0.061 & 0.75 \\
5850 & 5849.80 & 0.069 & 0.06 & 0.053 & 0.82 \\
6196 & 6195.96 & 0.084 & 1.5 & 0.90 & 0.69 \\
6284 & 6283.85 & 0.32 & 0.096 & 0.088 & 0.62 \\
6379 & 6379.29 & 0.10 & 0.29 & 0.21 & 0.80 \\
6614 & 6613.56 & 0.24 & 0.14 & 0.12 & 0.95 \\
6993 & 6993.18 & 0.14 & 0.29 & 0.25 & 0.99 \\
7224 & 7224.00 & 0.21 & & & \\
\hline
\end{tabular}

$A_{\mathrm{c}}=1-F\left(\lambda_{0}\right) / F$ (continuum).

The reference wavelengths are taken from Galazutdinov et al. (2000) (Col. 2). The equivalent width per extinction unit (this work) derived from published data from Jenniskens \& Désert (1994), Weselak et al. (2001), Thorburn et al. (2003) and Megier et al. (2005) is given for each DIB in Col. 5, with corresponding correlation coefficients in Col. 6 .

reddening excess in these stars must be circumstellar in origin. Other sources, however, show a relatively small reddening that is fully compatible with the values observed in field stars located at the same galactic latitude.

Based on this analysis, we divided our sample of post-AGB stars into two groups according to whether the overall observed

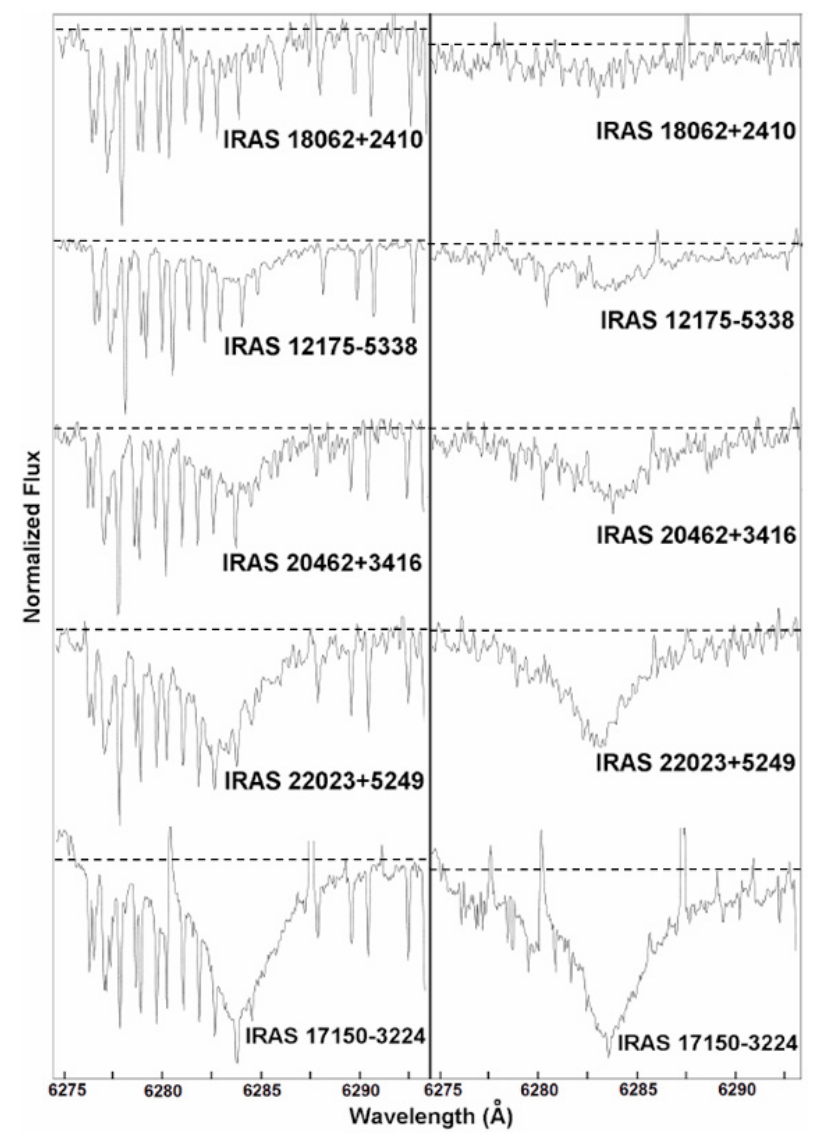

Fig. 3. Sample spectra showing the region around the DB at $6284 \AA$, before (left panel) and after (right panel) removal of the telluric lines using HD 172324 as the telluric divisor. Dotted lines indicate the continuum level adopted in each case.

extinction is more likely to be dominated by the circumstellar contribution (DCS/CS1-type stars) or just consistent with the interstellar extinction expected according to its Galactic latitude. This division is shown in Fig. 5.

Note that the above classification is very rough and that it only considers a star as belonging to the DCS group if it shows a relative large reddening excess with respect to the nominal value expected from its galactic location. Stars in which there is only a moderate (although possibly significant) contribution from the circumstellar shell to the observed extinction may have escaped detection. This means that the group of stars not classified in the DCS group may still contain sources in which the circumstellar contribution to the observed reddening is not negligible; and vice versa, those classified DCS may still contain a significant interstellar dust contribution.

To estimate an upper limit to the contribution of the interstellar reddening to the total reddening we use the Galactic 3D-extinction model map by Drimmel et al. (2003) that gives the mean visual extinction as a function of sky (galactic) coordinates and distance. The extinction has a projected resolution of $0.35^{\circ} \times 0.35^{\circ}$ (this is set by the COBE map used to re-scale the extinction so the model can reproduce correct far-infrared flux). It is evident that any small-scale structure (including the circumstellar contribution of the target star) is washed out in these estimates, which nevertheless give us information on the global spatial distribution of dust in specific directions. We take distance estimates from the literature where possible (Col. 3 in Table 4) and/or extract the maximum extinction (Col. 7) and the 

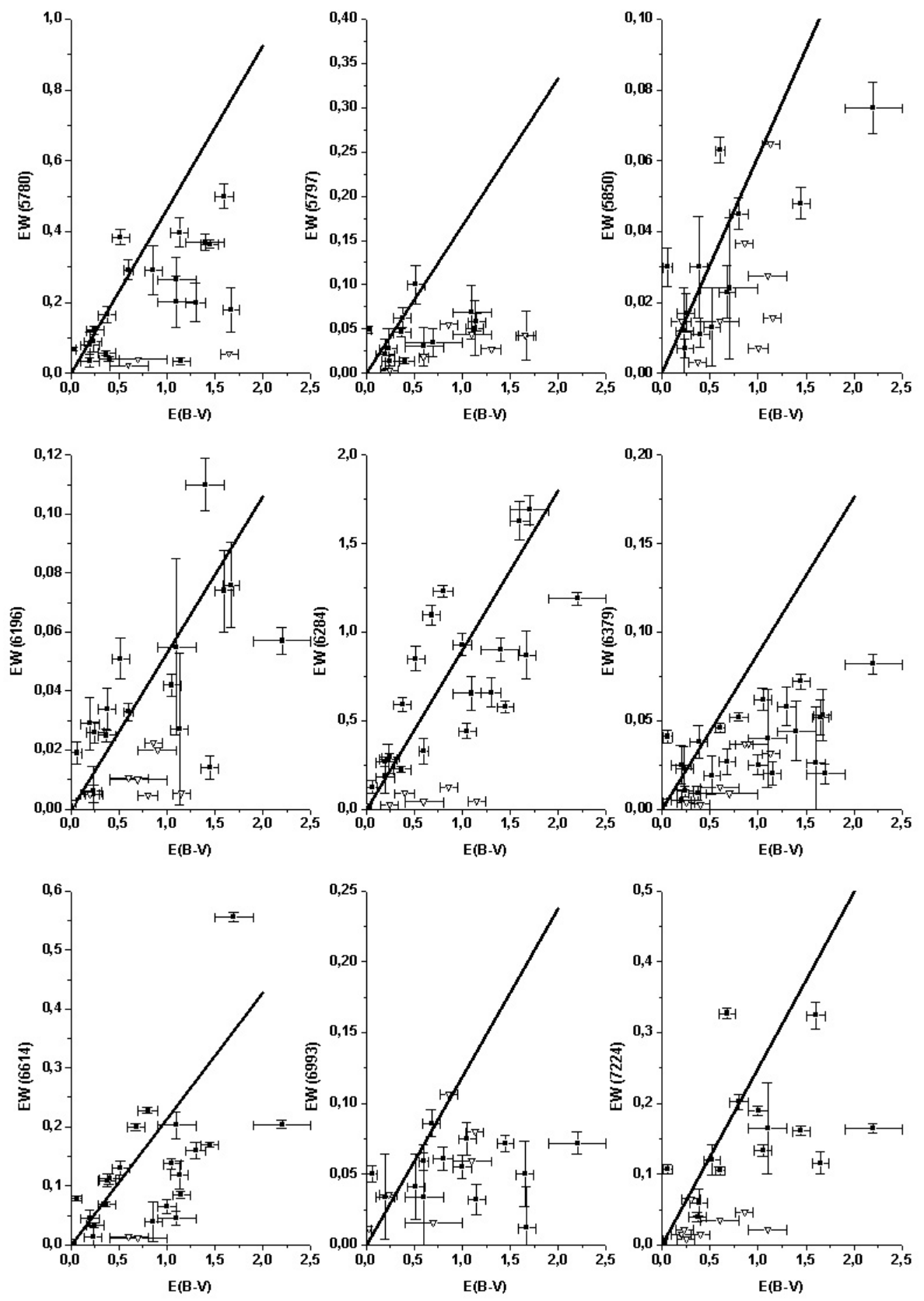

Fig. 4.Equivalent width (in $\AA$ ) of the 9 DBs selected for analysis as a function of $E(B-V)$ for the post-AGB stars in the sample. Solid lines correspond to the fits derived in this paper for field stars dominated by interstellar extinction (see also Sect. 4.1). The inverted triangles represent upper limits.

corresponding distance (Col. 6) for a particular line-of-sight. For high latitudes, the extinction versus distance curve flattens rapidly, within a few kpc. These (upper limit) estimates for the interstellar visual extinction, converted to reddening by dividing by the canonical value for $R_{V}=3.1$, can then be subtracted from the total observed reddening and thus yield (lower limit) estimates for the circumstellar reddening (Col. 9). Caution is needed when applying these results to derive extinction values for individual sightlines. Notwithstanding, we can now review and improve our (statistical) classification based on reddening versus latitude.

We consider all targets that are estimated to have a (lower limit) circumstellar reddening contribution higher than $50 \%$ of the total observed reddening to be of DCS-type (CS2 in Table 4). From the 11 DCS-type targets previously selected on the basis of their latitude reddening excess, we find that 9 satisfy this criterion. For the other 2 targets we can attribute a high fraction of the total observed reddening to interstellar dust.
Among the 22 IS (interstellar) reddening-dominated targets selected above via their latitude, we find 7 targets with a non-negligible circumstellar reddening $E(B-V)_{\mathrm{CS}} \geq$ 0.4 mag (Table 4; indicated CS2). IRAS 02229+6208 and IRAS 08544-4431 are special cases because these lines-ofsight show both strong interstellar and circumstellar dust contributions.

In Table 4 we give the extinction estimates derived from the model with rescaling of the spiral component (Col. 7). Norescaling estimates are given $(\mathrm{Col} .8)$ in those cases for which the extinction estimate is significantly higher than with use of rescaling. Distance estimates (references in Col. 4) and corresponding model extinctions (converted to $E(B-V)$ ) are given when available in Cols. 3 and 5). The maximum model reddening (with and without re-scaling) and the corresponding distance in the target direction are given in Cols. 7, 8 and 6, respectively. Column 9 gives the resulting lower limit for the circumstellar reddening. The final Col. 10 indicates when the target is 
Table 4. Line-of-sight properties for the observed post-AGB stars.

\begin{tabular}{|c|c|c|c|c|c|c|c|c|c|}
\hline \multirow[t]{2}{*}{ Name } & \multirow{2}{*}{$\begin{array}{l}E(B-V) \\
\text { observed }\end{array}$} & \multirow{2}{*}{$\begin{array}{l}d \\
\mathrm{kpc}\end{array}$} & \multirow[t]{2}{*}{ Ref. } & \multirow[t]{2}{*}{$\overline{E E(B-V) I S}$} & \multirow{2}{*}{$\begin{array}{l}d_{\max } \\
\mathrm{kpc}\end{array}$} & \multicolumn{2}{|c|}{$\overline{E E(B-V) \mathrm{IS}}$} & \multirow{2}{*}{$\begin{array}{l}E(B-V)-C S \\
\text { min }\end{array}$} & \multirow[t]{2}{*}{ 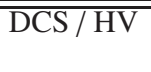 } \\
\hline & & & & & & scaling & no scaling & & \\
\hline $01005+7910$ & $0.2 \pm 0.1$ & 3 & & 0.11 & 1.7 & 0.11 & & & \\
\hline $02229+6208$ & $1.67 \pm 0.09$ & $>2.2$ & & 0.60 & 5 & 0.99 & & 0.68 & $\mathrm{CS} 2$ \\
\hline $04296+3429$ & $1.3 \pm 0.1$ & 3.5 & & 0.23 & 3.3 & 0.23 & & 1.07 & CS1 \\
\hline & & $5.4,5$ & 2,9 & 0.23 & & & & & \\
\hline $05113+1347$ & $1.1 \pm 0.2$ & 5 & 9 & 0.14 & 2.1 & 0.14 & & 0.96 & CS1 \\
\hline 05251-1244 & $0.23 \pm 0.09$ & & & & 1 & 0.18 & & & \\
\hline $05341+0852$ & $1.65 \pm 0.09$ & 10 & 9 & 0.16 & 2.4 & 0.16 & & 1.49 & CS1 \\
\hline $06530-0213$ & $1.7 \pm 0.2$ & 3 & 9 & 0.36 & 5 & 0.41 & & 1.29 & $\mathrm{CS} 2$ \\
\hline $07134+1005$ & $0.4 \pm 0.1$ & & & & 2.8 & 0.024 & 0.14 & $0.38 / 0.26$ & $\mathrm{HV} / \mathrm{CS} 2$ \\
\hline 08005-2356 & $0.7 \pm 0.3$ & & & & 4 & 0.18 & 0.27 & $0.52 / 0.43$ & $\mathrm{CS} 2$ \\
\hline 08143-4406 & $0.8 \pm 0.1$ & 4 & 9 & 0.55 & 5.8 & 0.66 & & & \\
\hline 08544-4431 & $1.45 \pm 0.09$ & & & & 6 & 0.99 & & 0.46 & CS2? \\
\hline $12175-5338$ & $0.25 \pm 0.09$ & & & & 3 & 0.24 & & & \\
\hline $16594-4656$ & $2.2 \pm 0.3$ & 2.6 & 10,11 & & 7 & 2.1 & & & $\mathrm{CS}(*)$ \\
\hline $17086-2403$ & $0.86 \pm 0.09$ & 6 & 9 & 0.56 & 2 & 0.56 & & 0.3 & HV (CS2?) \\
\hline $17097-3210$ & $0.06 \pm 0.05$ & $(0.2)$ & & $(0.08)$ & 5 & 0.95 & & & \\
\hline $17150-3224$ & $0.68 \pm 0.09$ & $<18(2)$ & 9 & $(0.9)$ & 5 & 1.8 & & & \\
\hline $17245-3951$ & $1.0 \pm 0.1$ & & & & 5 & 1.0 & 1.5 & & HV \\
\hline $17395-0841$ & $1.1 \pm 0.2$ & & & & 2 & 0.87 & & 0.23 & $\mathrm{HV}$ \\
\hline $17423-1755$ & $1.13 \pm 0.09$ & $3.2-3.7$ & & 0.6 & 4 & 0.60 & & 0.53 & CS1 / HV \\
\hline $17436+5003$ & $0.24 \pm 0.09$ & 1.2 & & 0.03 & 0.9 & 0.026 & 0.064 & 0.18 & CS1 \\
\hline $18025-3906$ & $1.15 \pm 0.09$ & & & & 2 & 0.23 & 0.34 & $0.92 / 0.81$ & CS1 / HV \\
\hline $18062+2410$ & $0.6 \pm 0.2$ & $4.5-5.3$ & & 0.14 & 1.4 & 0.14 & & 0.46 & CS1 / HV \\
\hline HD 172324 & $0.03 \pm 0.02$ & & & & 1.5 & 0.056 & 0.11 & & \\
\hline $19114+0002$ & $0.60 \pm 0.05$ & 1.5 & 4 & 0.32 & 5 & 0.54 & 0.62 & & $\mathrm{HV}$ \\
\hline $19200+3457$ & $0.3 \pm 0.1$ & & & & 2.9 & 0.15 & 0.22 & & \\
\hline $19386+0155$ & $1.05 \pm 0.09$ & & & & 2.9 & 0.35 & & 0.80 & CS1 \\
\hline 19500-1709 & $0.37 \pm 0.09$ & $>4$ & 12 & & 1.0 & 0.20 & & & \\
\hline $20000+3239$ & $1.6 \pm 0.1$ & $(5,8)$ & & $(1.0,1.7)$ & $>10$ & $>1.7$ & & ? & \\
\hline $20462+3416$ & $0.38 \pm 0.09$ & & & & 4 & 0.25 & 0.38 & & HV \\
\hline $22023+5249$ & $0.52 \pm 0.09$ & $3.3-3.9$ & & $0.47,0.50$ & 5 & 0.52 & 0.55 & & $\mathrm{HV}$ \\
\hline $22223+4327$ & $0.2 \pm 0.1$ & & & & 2.6 & 0.21 & & & \\
\hline $22272+5435$ & $0.9 \pm 0.2$ & $1.6,2.7$ & 8 & $0.26,0.39$ & 4.5 & 0.47 & & 0.43 & $\mathrm{CS} 2$ \\
\hline $23304+6147$ & $1.4 \pm 0.2$ & $4.7,5$ & 5,9 & 0.64 & 4.5 & 0.64 & & 0.76 & $\mathrm{CS} 2$ \\
\hline
\end{tabular}

DCS = dominated by circumstellar reddening; HV = high radial velocity; CS1 designated DCS based on lat/long vs. reddening, excluding those that are discarded based on interstellar (IS) reddening estimate. CS2 indicates DCS found by estimating the upper limit to the IS reddening.

[1] Reddy et al. (1999); [2] Klochkova et al. (1999); [3] García-Lario et al. (1999); [4] Zacs et al. (1999a,b); [5] Klochkova et al. (2000); [6] Bakker et al. (1997); [7] Hrivnak \& Kwok 1991; [8] Woodsworth et al. (1990); [9] Reddy \& Parthasarathy (1996); [10] Hrivnak et al. (2000); [11] van de Steene \& van Hoof (2003); [12] Clube \& Gledhill (2004), $A(V)=1.2$. (*) IRAS 16594-4656 is a well known bi-polar PPN which is thought to have a high internal extinction (see also Sect. 4.3).

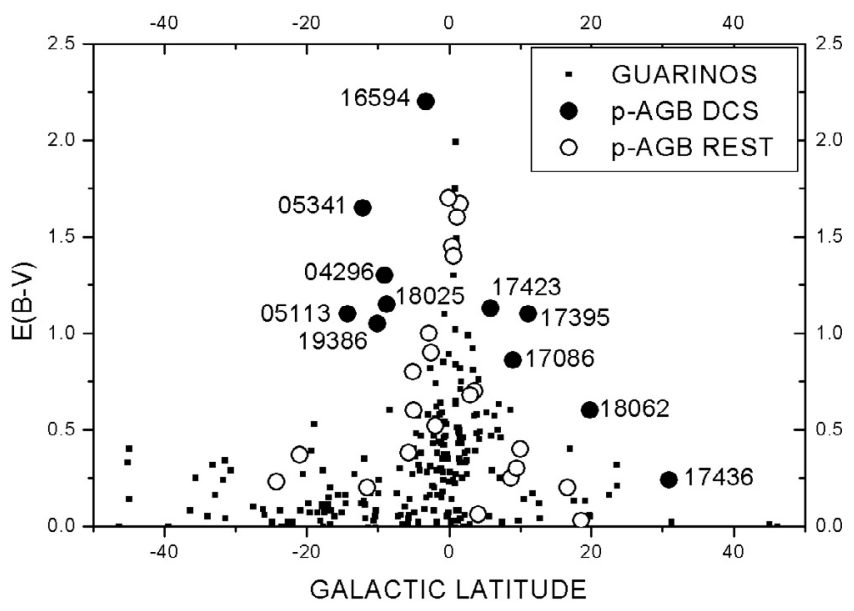

Fig. 5. $E(B-V)$ versus Galactic latitude distribution of the post-AGB stars in the sample (circles) and for reference stars taken from Guarinos (1988a,b, 1997) (small squares). Post-AGB stars dominated by circumstellar extinction (DCS-type) are indicated by filled circles and are labelled with their IRAS name. dominated by circumstellar reddening (CS1 or CS2) and/or is a high velocity target (HV).

\subsection{DB strength in DCS-type post-AGB stars}

Following the above criteria, we find that for 17 (out of 33) stars in the sample a significant fraction of the observed reddening is due to the presence of circumstellar dust (i.e. DCS-type), while for the remaining targets the colour excesses are expected to be predominantly due to interstellar dust.

A comparison of the DB strengths measured in stars belonging to each of the two groups considered above with those found in reference field stars is presented in Fig. 6, where we can see that there is a general trend for the stars with a dominant circumstellar extinction contribution (DCS-type) to show much weaker DB strengths compared to the rest of post-AGB stars in the sample. In the most extreme cases, there are stars in this group affected by a large overall extinction in which surprisingly some DBs are completely absent, and only upper limits to their equivalent width can be reported. In contrast, we find a significant number of sources among the rest of post-AGB stars in the sample 

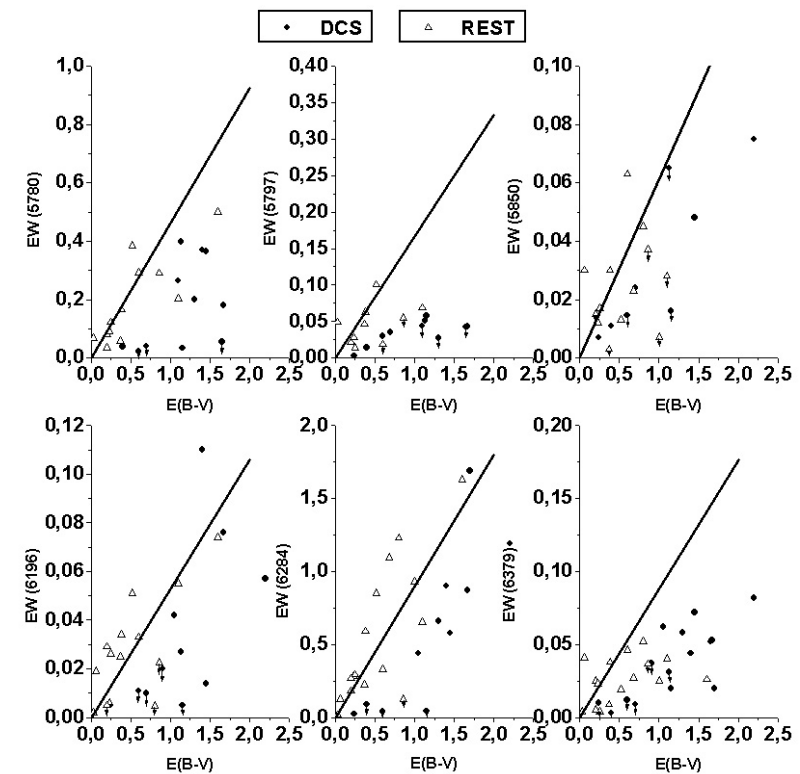

(
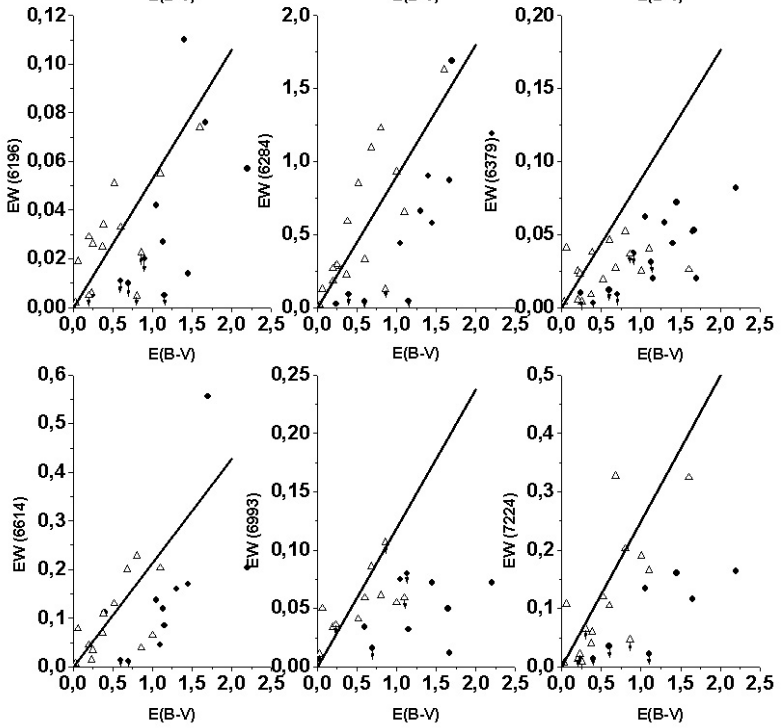

$\mathrm{E}(\mathrm{B}-\mathrm{V})$

E(B-V)

Fig. 6. Equivalent width of the 9 DBs selected for analysis as a function of $E(B-V)$ for the post-AGB stars in the sample in which filled circles represent the subsample of post-AGB stars dominated by circumstellar extinction (DCS-type) and open triangles the rest of stars in the sample. Solid lines again correspond to the fits derived in this paper for field stars dominated by interstellar extinction. The arrows indicate upper limits.

showing DB strengths fully in agreement with those observed in field stars.

In many cases DBs are observed towards the DCS-type stars (Figs. 7 to 15; left panels). In order to assess the circumstellar contribution, the observed $E W$ can be corrected by subtracting the expected DIB $E W$ found by applying the estimated interstellar reddening (Table 4$)$ to the respective $E W / E(B-V)$ for field stars (Table 3 ), as well as by subtracting the IS reddening contribution from the total observed reddening. The introduced uncertainties are quite large due to the scatter on the derived linear relationships (see above, Sect. 4.1). In particular IRAS 02229+6208 and IRAS 08544-443 require a correction for significant IS dust.

In the other direction, we can correct the IS-type stars (left panel of Figs. 7 to 15 ) by subtracting the CS (circumstellar) reddening contribution from the total reddening and the circumstel$\operatorname{lar} E W / E(B-V)$ (which we assume to be zero) from the observed $E W$. It then shows that all stars coincide neatly with the average Galactic relation. This is most noticeable for IRAS 17086-403 and IRAS 17395-0841, which have estimated CS contributions of 0.3 and $0.2 \mathrm{mag}$, respectively).

In principle, this result supports our initial interpretation that the DB carrier(s) may not be present in the circumstellar envelopes of post-AGB stars. However, strong variations from source to source are still visible in both groups of stars, which may be related to other observational properties of those shells not yet considered. (Significant scatter is also observed for the sample of field stars; Sect. 4.1.) Indeed, the results suggest that some of the DB carriers could be completely absent in some of these envelopes while not in others.

\subsection{DB strength vs. chemistry and spectral type}

To explore whether other environmental conditions, like the dominant chemistry in the shell or the spectral type of the central star could also play a role in the differences observed between individual stars in the sample, we have divided the two groups defined above further into another four subgroups as a function of whether the chemistry of the shell is carbon-rich or oxygenrich, or the spectra of the central star is early-type $(B-A)^{5}$ or intermediate-type (F-G).

We do this because the dominant chemistry of the shell can determine the formation of specific compounds in the circumstellar shell completely. In oxygen-rich shells, we expect to find aluminum oxides, amorphous, or crystalline (fayalite, enstatite, forsterite, etc.) silicates, water ice, and other main constituents of oxygen-rich dust grains. In carbon-rich stars, instead, we can find carbon-based constituents, like chains or rings of carbon, graphite, hydrogenated amorphous carbon grains, fullerenes, nanodiamonds, or PAHs.

If a DB carrier had its origin in a compound or constituent related to only one of the above chemistries, we would expect to observe differences in strength from source to source as a function of their particular chemical composition. On the other hand, it is also well known that the UV radiation field plays a crucial role in processing the circumstellar dust grains, not only immediately after they are formed, while they are still part of the shell, but also later when they are released to the ISM. Dust grains in the circumstellar envelopes of post-AGB stars are exposed to increasing doses of UV radiation due to the increasing effective temperature of the central star during its fast evolution towards the planetary nebula stage. First, when the central star is still showing late-to-intermediate spectral type, the UV radiation can be neglected, both as a consequence of the low effective temperature of the central star and because the higher density in the envelope during the early post-AGB stage would effectively protect (at least temporarily) circumstellar dust grains from the energetic UV photons coming from the ISM. These conditions favour the formation of large dust grains that can survive in this less aggressive environment. Later in the post-AGB evolution, the central stars become early-type and start producing a considerable number of UV photons that may lead to an efficient processing of the dust grains in the shell, which in turn is less protected and also more vulnerable to the UV radiation field coming from the ISM. The combined effect of the UV photons coming from the central star and from the ambient ISM is expected to accelerate the processing of the dust grains, leading to new species like molecules, radicals (more or less complex), and other byproducts resulting from the partial or total evaporation of the grains, which will eventually be released to the ISM. Indeed, these byproducts could be the actual carriers of the DIBs commonly observed in the ISM.

If DB carriers were only related to the byproducts of the decomposition of these large circumstellar grains, we would expect to observe a deficit in DB strengths in post-AGB stars, but only

\footnotetext{
5 Note that among the subgroup of early-type stars we have also included the few sources in Table 2 that are classified as planetary nebulae.
} 

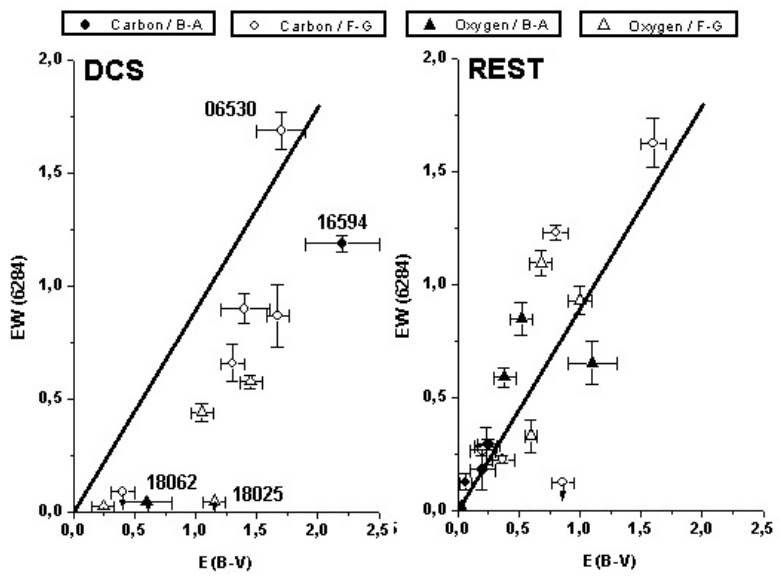

Fig. 7. Equivalent width of the $6284 \AA$ band vs. $E(B-V)$ for the group of stars dominated by circumstellar extinction (left panel; DCS type) and for the rest of stars in the sample (right panel) with different symbols indicating the dominant chemistry and spectral type of the observed stars. The solid line represents the behaviour observed in field stars dominated by interstellar extinction, as deduced from the data shown in Fig. 2.
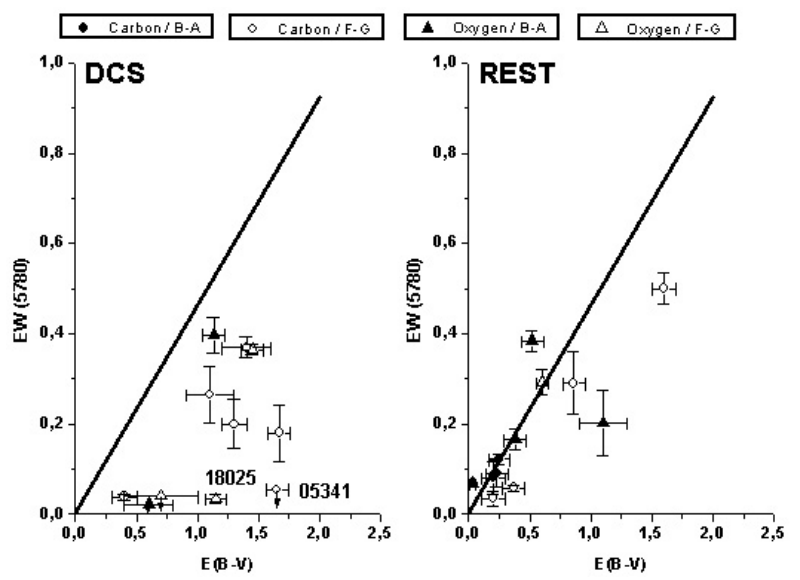

Fig. 8. Same as Fig. 7, for the $5780 \AA$ band.

while the central stars are still showing a relatively low effective temperature. Both effects can be combined, and it may also happen that the DB carriers are related to the byproducts of only a particular class of grains associated to a given dominant chemistry. In this case, we will be able to detect significant differences from source to source, as a function of both the spectral type of the central star and of the dominant chemistry in the shell. In the next section we analyse the influence of these environmental conditions (dominant chemistry and spectral type) on the observed results for each of the 9 DBs under study in our sample of post-AGB stars.

\subsection{Analysis of individual bands}

\subsubsection{Analysis of the $6284 \AA$ band}

The $6284 \AA$ band is not only the strongest $(E W / E(B-V)=$ $0.90 \AA / m a g$ in the ISM) but also the broadest band included in our analysis. As such, it is relatively easy to measure, in spite of the contamination by telluric lines already shown in Fig. 3, which must be carefully removed.

In Fig. 7 we show the results obtained as a function of the dominant chemistry and of the spectral type of the central star for each of the two main subgroups identified in our sample.
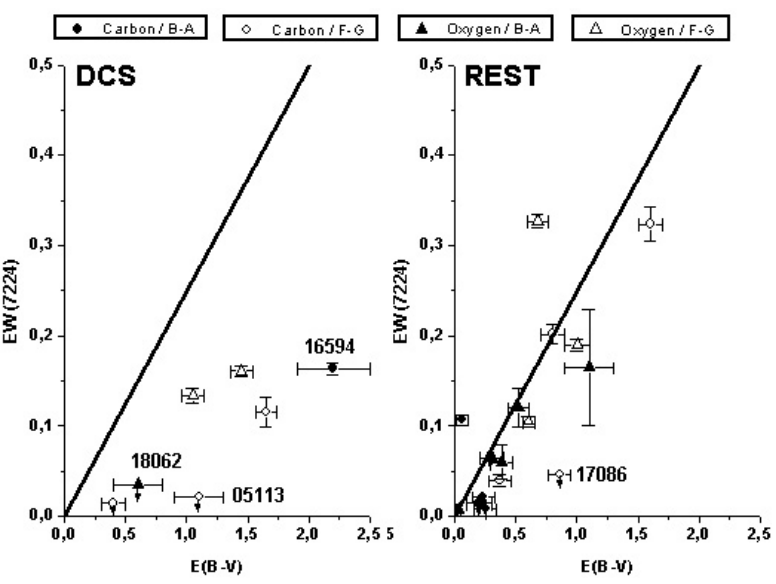

Fig. 9. Same as Fig. 7, for the $7224 \AA$ band.
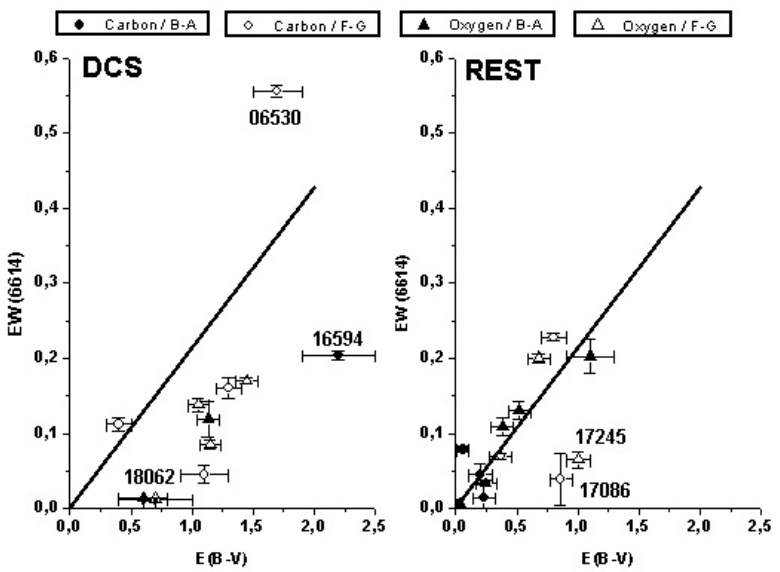

Fig. 10. Same as Fig. 7, for the $6614 \AA$ A band.
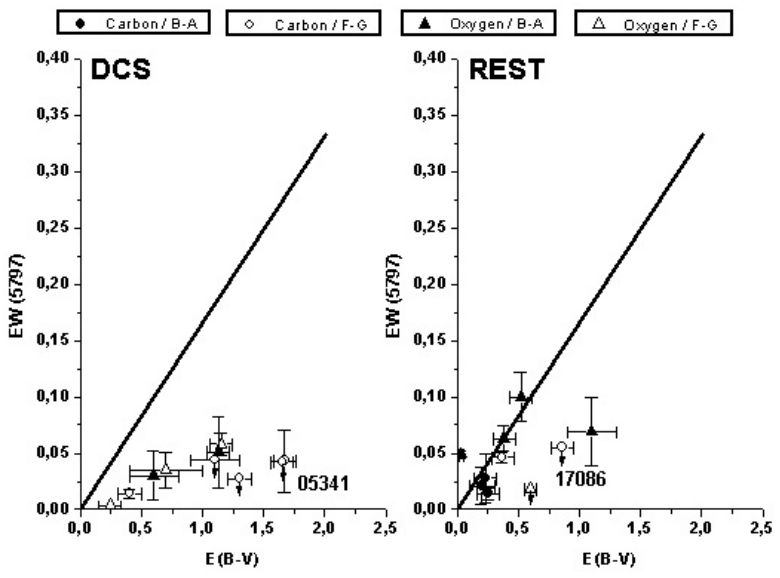

Fig. 11. Same as Fig. 7, for the $5797 \AA$ A band.

The strength of the $6284 \AA$ band as a function of $E(B-V)$ for the group of stars dominated by circumstellar extinction is presented in the left panel, while the results obtained for the rest of stars in our sample is shown in the right panel. As we can see, it is obvious that the post-AGB stars belonging to the DCS group show DB strengths systematically below those observed in the ISM.

Actually, in some cases this band is so weak that we can only determine an upper limit for its equivalent width. This is the case for IRAS 17436+5003 (oxygen-rich; F type), IRAS 18025-3906 

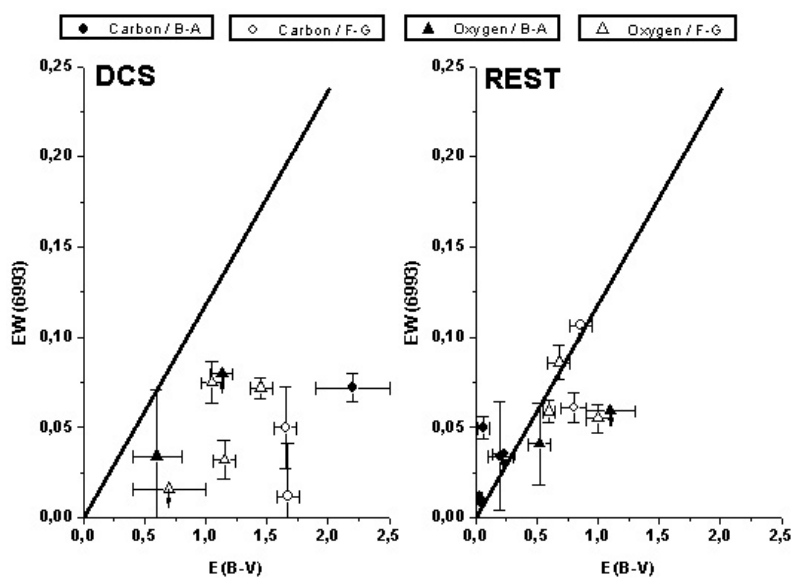

Fig. 12. Same as Fig. 7, for the $6993 \AA$ band.

(oxygen-rich; G type), and IRAS 18062+2410 (oxygen-rich; B type). It must be noted that the quality of the available spectra for these four stars is good enough to discard a non-detection that could be attributed to a poor signal-to-noise ratio.

Even for IRAS 16594-4656 (carbon-rich; B-type), the star with the second strongest feature in our sample, we find that the $6284 \AA$ band is a factor of two weaker than expected for its high value of $E(B-V)$. This star is a well-known bipolar proto-planetary nebula that seems to be affected by a high internal extinction.

For IRAS 06530-0213, the band strength is typical of the total observed reddening being due to interstellar dust. On the other hand, the interstellar and circumstellar reddening contributions are estimated to be 1.3 and $0.4 \mathrm{mag}$, respectively (Sect. 4.3). Though this could indicate the presence of circumstellar DBs, it should be noted that this line of sight lies in the galactic plane (GLAT $=-0.13$ degrees) and the interstellar reddening could be underestimated by the extinction model.

Remarkably, we find stars with a very weak band in all the subgroups, irrespective of the dominant chemistry and spectral type considered. This almost completely rules out the possibility of the $6284 \AA$ band being generated in the circumstellar envelope of post-AGB stars, at least in the same proportion as in the ISM. In contrast, in the right panel we can see that in general the rest of stars in the sample show a trend that is very similar to the one observed in the field stars in which the extinction is dominated by the interstellar contribution.

\subsubsection{Analysis of the $5780 \AA$ band}

This DB has an $E W / E(B-V)=0.46 \AA / \mathrm{mag}$ in the ISM, so it is the second most intense one after the $6284 \AA$ band. In this particular case, it is important to note that the spectral region corresponding to this band can be contaminated by the presence of photospheric lines, which makes evaluation of the band strength very difficult in stars with intermediate and late spectral types.

Note that to distinguish weak features from weak stellar lines or telluric contaminations is not always a simple task, and this makes it necessary to use detailed stellar models (to subtract the atmospheric features) and high-resolution spectroscopy (to properly remove undesired contaminations), as the only way to derive the accurate strength of the band, which is beyond the scope of this work.

In Fig. 8 we show the equivalent width of the $5780 \AA$ band vs. $E(B-V)$ for each of the two main subgroups in which we
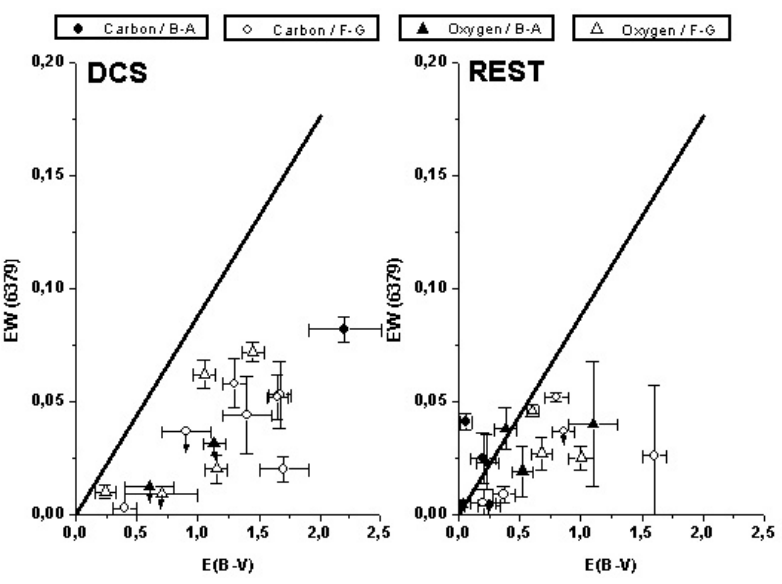

Fig. 13. Same as Fig. 7, for the $6379 \AA$ A band.
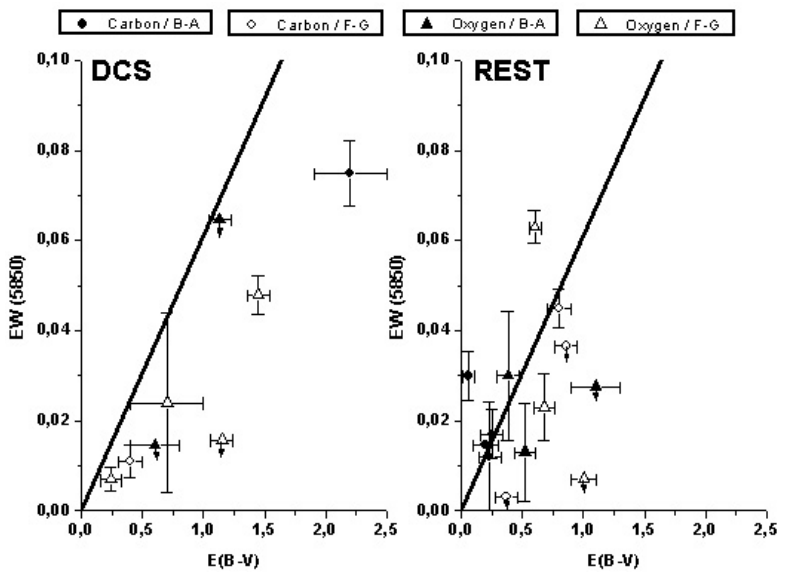

Fig. 14. Same as Fig. 7, for the $5850 \AA$ band.
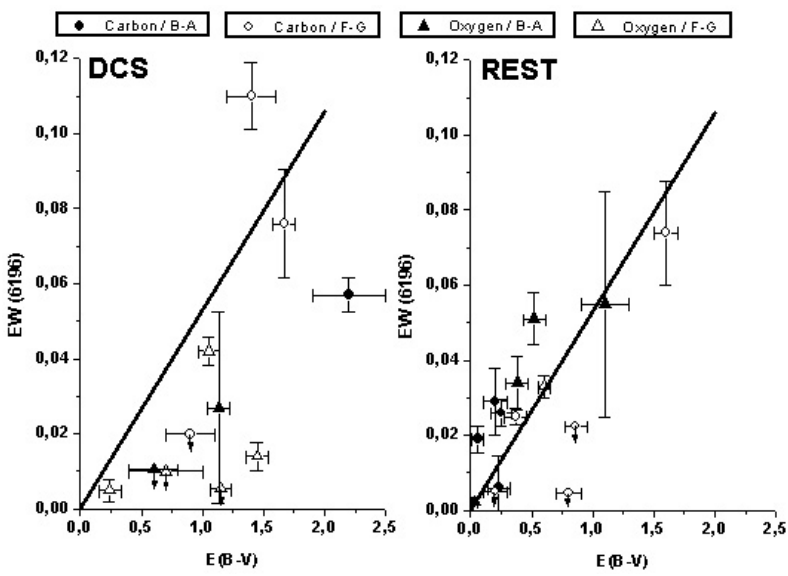

Fig. 15. Same as Fig. 7, for the $6196 \AA$ band.

have divided the sample. In the group of stars dominated by circumstellar extinction (left panel), we again observe strengths significantly weaker than those expected in stars for which the extinction is mainly of interstellar origin.

The non-detection of this DB in IRAS $05341+0852$ (carbonrich; F-type), despite the high value of $E(B-V)_{\mathrm{CS}} \approx 1.5 \mathrm{mag}$, is remarkable. This clearly suggests that the carrier of this band is completely absent at least in the envelopes of carbon-rich stars with intermediate spectral types. A similar conclusion can be derived for oxygen-rich stars with intermediate spectral types from 


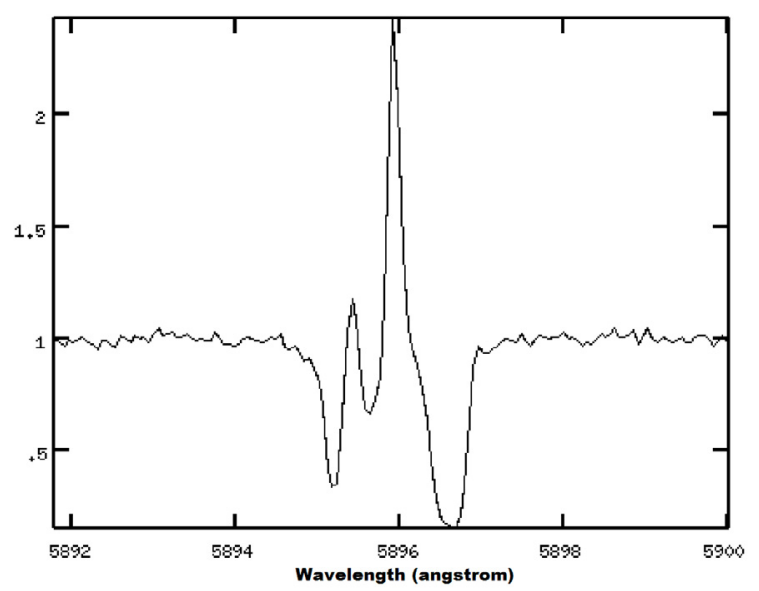

Fig. 16. The complex profile of the $\mathrm{Na}_{\mathrm{I}} \mathrm{D}_{1}$ line, as observed in IRAS $04296+3429$.

the very weak strength observed in IRAS 18025-3906 (oxygenrich; B-type).

Unfortunately, the spectrum available for IRAS 16594-4656 does not cover the spectral range corresponding to this band, so we cannot extend the above conclusion to carbon-rich stars with earlier spectral types based on our data.

As in the case of the $6284 \AA$ band, we can also observe in the right panel of Fig. 8 that the rest of stars in the sample not identified as dominated by circumstellar extinction show a position in the diagram that does, overall, agree more with the results obtained for field stars in which the extinction is mainly of interstellar origin.

\subsubsection{Analysis of the $7224 \AA$ band}

This DB is not usually analysed in the literature because it is strongly affected by telluric contamination. As in the case of the $6284 \AA$ band, we have carefully eliminated this contribution by dividing the normalised spectrum by the spectrum of the unreddened target HD 172324 (see Sect. 2).

In Fig. 9 we show the equivalent width of the $7224 \AA$ band vs. $E(B-V)$ for each of the two main subgroups identified in our sample. As for the two previous features, we find strengths that are much weaker than those measured in field stars in the subgroup formed by the stars in which the circumstellar contribution to the overall extinction is dominant (DCS; left panel). This again suggests that this band is not formed in the circumstellar envelope of post-AGB stars.

Again, the measured intensity of the $7224 \AA$ band in IRAS 16594-4656 is rather weak and, once more, we find several non-detections: IRAS 05113+1347 (carbon-rich star) and IRAS $18062+2410$. The latter case is remarkable because this is an oxygen-rich star with a B spectral type, where we have not detected the $6284 \AA$ band. The non-detection of DBs in oxygen-rich envelopes around post-AGB stars indicates that the DB carriers are probably not generated in oxygen-rich environments. The absence of the band in the specific case of IRAS $18062+2410$ suggests that they are not produced even when the central star temperature is hot enough to produce high levels of UV irradiation on the (oxygen-rich) circumstellar grains.

For the rest of stars in the sample (Fig. 9; right panel), we again find a behaviour that seems to closely follow the general trend observed in reference stars affected only by interstellar

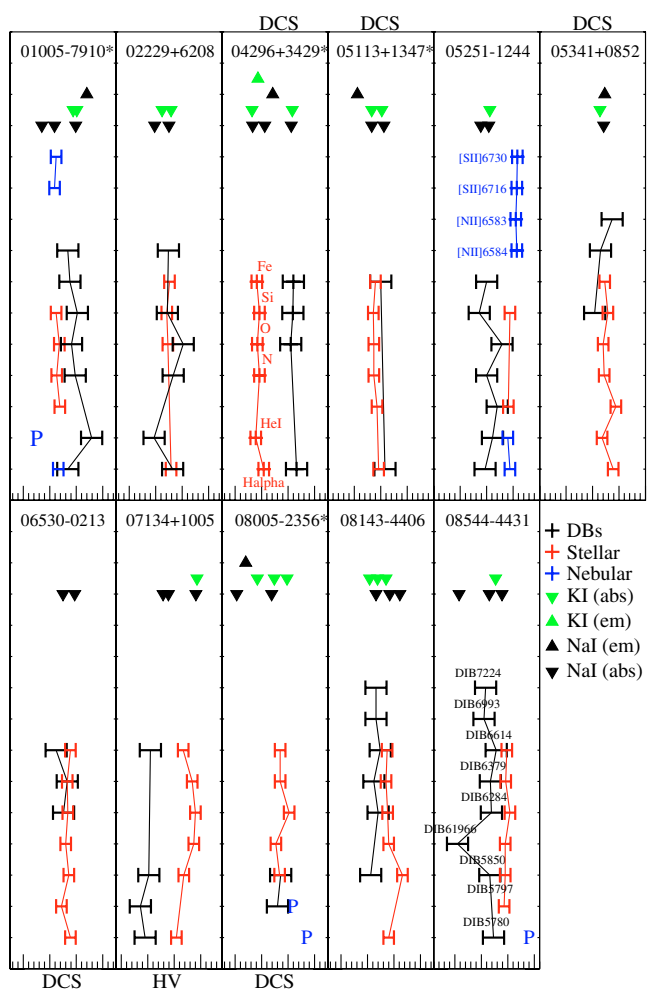

Fig. 17. Velocities of the DBs and the stellar and interstellar absorption lines and the nebular emission line components plotted per panel for each target. The $y$-axis is in arbitrary units. The $x$-axis is the LSR velocity in $\mathrm{km} \mathrm{s}^{-1}$, with big ticks separated by $50 \mathrm{~km} \mathrm{~s}^{-1}$. Note that the widths of the panels are identical, i.e. $200 \mathrm{~km} \mathrm{~s}^{-1}$, but the central velocity of each is shifted to show all lines for each target. Error bars are $\leq 10 \mathrm{~km} \mathrm{~s}^{-1}$ for stellar lines, $\leq 20 \mathrm{~km} \mathrm{~s}^{-1}$ for DBs, and $\leq 5 \mathrm{~km} \mathrm{~s}^{-1}$ for the sodium and potassium components. Targets dominated by circumstellar reddening are labelled "DCS" and high radial velocity targets are labelled "HV" The stellar and DB velocity components can be compared directly to those of neutral sodium and potassium in either emission (upward arrow) or absorption (downward arrow) plotted at the top of each panel.

extinction. The exception is IRAS 17086-2403 (carbon-rich star) for which we detect no DBs.

\subsubsection{Analysis of the $6614 \AA$ band}

In Fig. 10 we show the results of our analysis applied this time to the $6614 \AA$ band. In the left panel we show the equivalent widths measured in the subgroup of post-AGB stars dominated by circumstellar extinction. As for the other bands, we see strengths that are systematically weaker than in the reference stars dominated by interstellar extinction. The only non-detection in this case corresponds to IRAS $18062+2410$, which we have previously pointed out as non-detected in the analysis made for the bands centred at 6284 and $7224 \AA$. The weak strength of this DB in IRAS 16594-4656 (carbon-rich, B type) is again found to be compatible with the absence of its carrier in the circumstellar envelope of this star, and it confirms that the overall extinction affecting this source is the result of a quite similar contribution from the ISM and from the circumstellar material. The $6614 \AA$ DB detected toward IRAS 06530-0213 is unusually strong for DBs in the DCS group and even with respect to the Galactic relationship. As mentioned earlier for the $6284 \AA$ band toward the same target, this could point, for this particular source, towards 

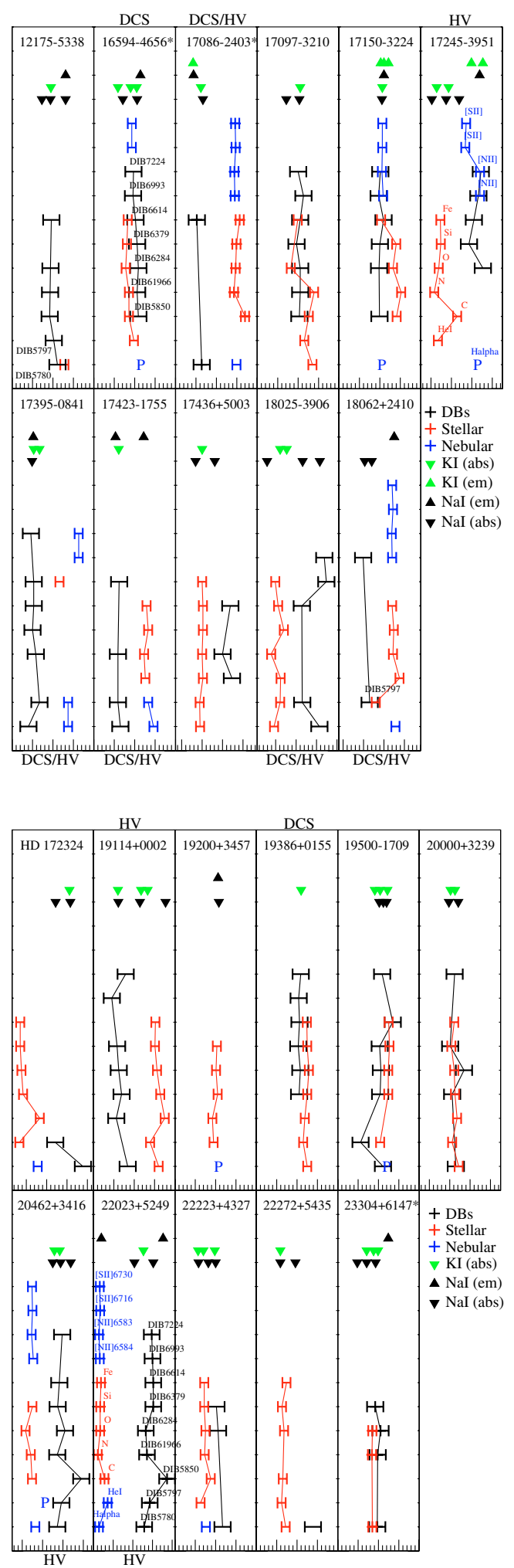

Fig. 16. continued.

the presence of circumstellar DBs or, perhaps more likely, to an underestimation of the interstellar reddening.

For the rest of stars (right panel), as usual, we find that most of them are located in the region of the diagram corresponding to the field stars dominated by interstellar extinction. In this case, we would like to point out only the slightly discrepant position occupied by the oxygen-rich, F-type star IRAS 17245-3951

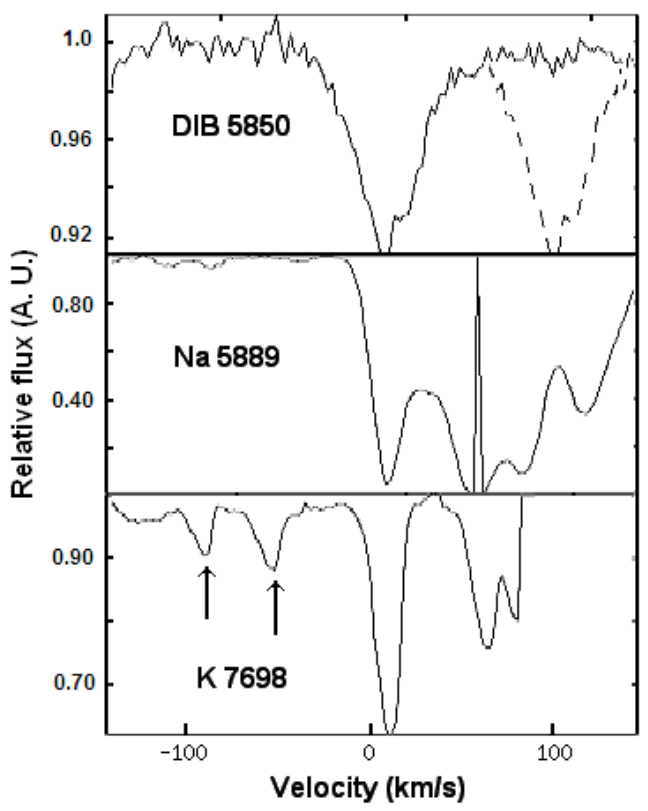

Fig. 17. The $5850 \AA$ band observed in the high radial velocity $\left(\sim 100 \mathrm{~km} \mathrm{~s}^{-1}\right)$ sample star IRAS 19114+0002 (solid line). The nondetection of this feature at the Doppler-shifted velocity corresponding to the central star (dashed line) confirms the interstellar nature of the band and the absence of a circumstellar component. The potassium and sodium velocity structures are shown in the bottom panels to illustrate the distribution of interstellar medium in this line of sight. A narrow sodium emission line is seen in the middle panel. The two arrows in the bottom most panel indicate two telluric features next to the potassium line.

not yet previously identified as an outlier in the above discussion. Again, the carbon-rich star IRAS 17086-2403 shows very weak DBs.

\subsubsection{Analysis of the $5797 \AA$ band}

This DB has been included in numerous studies in the literature because of its proximity to the nearby $5780 \AA$ band. This has allowed a comparative analysis of their relative intensities in different astrophysical environments.

The $5797 \AA$ band has a lower sensitivity to the extinction $E W / E(B-V)=0.17 \AA / \mathrm{mag}$ when it was measured in the ISM, compared to the previous DBs. Similar to the adjacent $5780 \AA$ band, it is necessary in our analysis to take into account the possible contamination due to the presence of atmospheric stellar lines in this spectral range in stars of intermediate and late spectral types, as it can affect our measurements.

In Fig. 11 we show the equivalent width of this band vs. $E(B-V)$ as it was measured from the available spectra for each subgroup of stars in which we divided the sample. For stars in the DCS group (left panel), all post-AGB stars are found to show DB strengths that are considerably weaker than in the field stars, which consistent with the results found in the other bands analysed so far.

Among the non-detections, we emphasise IRAS 05341+0852 (carbon-rich, $F$ type) taking its large extinction $E(B-V)_{\mathrm{CS}}=1.5 \mathrm{mag}$ into account. We recall that this star was also found to show no indication of the accompanying feature at $5780 \AA$. Unfortunately, the spectrum of IRAS 16594-4656 does not cover the wavelength corresponding to this DB (as happened with the $5780 \AA$ band). In the right 
panel (rest sample) the line-of-sight toward IRAS 17086-2403 again shows a conspicuous absence of DBs.

\subsubsection{Analysis of the $6993 \AA$ band}

This band is also among the ones not usually analysed in the literature, most likely because of the presence of telluric lines in the spectral range adjacent to this band but also because of the intrinsic weakness of this $\mathrm{DB}$, for which $E W / E(B-V)=$ $0.12 \AA / \mathrm{mag}$ in the ISM. In Fig. 12 we show the results of our analysis for this DB for each of the subgroups in which we have divided the sample, again as a function of the dominant chemistry and the spectral type of the central star.

The results are once more consistent with previous analyses of other DBs. We find a better agreement with the values obtained in reference stars dominated by interstellar extinction for the sources in the right panel, although in this case the effect is not as evident as in the previous analysis due to the larger errors associated to the measurements. Consistent results, although more sensitive to measurement errors, are obtained when the features at 6379, 5850, and $6196 \AA$ are analysed (see Figs. 13-15).

\subsection{Radial velocity analysis}

An additional way to check whether our conclusions are consistent with the available observational data is to analyse the Doppler velocities associated to the DBs detected in our stars.

The overall strategy consists of comparing these Doppler velocities with the radial velocities associated either to the atmospheric stellar absorptions or to the nebular and recombination emission lines sometimes detected in our spectra. In general, atmospheric and nebular lines are expected to match each other within the errors unless the central star is part of a binary system or the nebular shell shows a complex morphology.

If the DBs detected are formed in the circumstellar envelopes of these stars, we should measure Doppler velocities in these bands consistent with the characteristic radial velocities derived from the absorption and/or emission lines identified in the stellar spectra.

In Table 6 (online only) we give the radial velocities (in $\mathrm{km} \mathrm{s}^{-1}$ ), measured with respect to the local standard of rest (LSR) associated to several atoms and ions, as derived from various atmospheric stellar absorptions and nebular emission lines identified in the stars of our sample. In addition, we also display the measurements made in $\mathrm{H} \alpha$ at $6563 \AA$ and in the He I line at $6678 \AA$. In the case of the atmospheric absorption lines shown in Table 6, the average velocity derived from several line measurements corresponding to various ions of the elements $\mathrm{C}, \mathrm{N}$, $\mathrm{O}, \mathrm{Si}$, and $\mathrm{Fe}$ is presented. For the nebular lines, we only considered the forbidden lines of [N II] and [S II], found around $\mathrm{H} \alpha$. The typical uncertainties are of the order of $5-10 \mathrm{~km} \mathrm{~s}^{-1}$.

In addition, in Table 7 (online only) we present the velocities derived from the analysis of the Na I D (5889.95 and 5895.92 A) doublet and of the KI (7698.97 $\AA$ ) line, which are in most cases also clearly detected in our spectra (uncertainties are $\sim 3$ to $5 \mathrm{~km} \mathrm{~s}^{-1}$ ). These lines, like the DBs that we want to analyse, usually originate in the ISM, but they can also form in the circumstellar shell. In this case, the circumstellar component usually appears in emission over the interstellar absorption (see Fig. 16). In general, these lines show very complex absorption profiles as they reflect the different velocities of the clouds located along the line of sight. In some of our stars the circumstellar component may contribute significantly to the observed profile and can be used as a further test to identify the origin of analogue velocity components that may be present in our favourite DB. Table 8 (online only) shows the radial velocities associated to the DBs observed in the stars of our sample, which can then be compared to the velocities provided in Tables 6 and 7.

It is important to take into account that deriving velocities for DBs is in many cases a complicated task, especially if the features under analysis are weak in strength. In general, the Doppler shift measurements are determined by assuming that the absorption peak is a good approximation to the centre of the feature. We estimate that, on average, the errors in Table 8 may be affected by errors of the order of $10-20 \mathrm{~km} \mathrm{~s}^{-1}$.

Comparing Tables 6 and 7, we observe that in most cases there is at least one velocity component associated to the sodium doublet or the potassium line, either in emission or in absorption, that can be interpreted as having a stellar or circumstellar origin. The circumstellar nature of these lines is easy to determine when they are found in emission. The radial velocities measured in this case are usually coincident with the systemic velocity of the post-AGB star. There are a few cases that show a very complex $\mathrm{Na}$ I D line profiles in which the circumstellar emission appears superimposed on the interstellar absorption preventing a proper assignment (indicated in Table 7 and Fig. 17).

Figure 17 shows for comparison the velocities of the DBs and the (inter-)stellar absorption and emission components for each target. For the majority of the targets, these graphs show consistent velocities for the stellar lines. The DB velocities are also consistent with each other. For several cases the nebular (emission) lines are significantly shifted with respect to the atmospheric lines (e.g. IRAS 17245-3951) due to the binarity of the system and/or a complex wind structure. These stellar and DB velocity components can be compared directly to those of neutral sodium and potassium in the respective line-of-sight.

If DB carriers are present in the circumstellar envelopes of some of the post-AGB stars in our sample, we would also expect to find matches between the velocities shown in Table 8 and those in Table 6 (see Fig. 17), especially for those stars in which we detected circumstellar Na I in emission belonging to the DCS group. Remarkably, in no case did we find values consistent with the velocities associated to the DBs that cannot be explained as a natural consequence of interstellar clouds with a similar velocity present in the line of sight.

The inconsistency between velocities is more obvious if we have a look at those stars showing very high radial velocities (HV in Table 4 and Fig. 17). Several of the HV targets have radial velocities higher than $100 \mathrm{~km} \mathrm{~s}^{-1}$. Such high velocity differences are comparable to those measured for successfully detected extra-galactic DBs (Ehrenfreund et al 2002; Cox et al. 2007).

In the top panel of Fig. 17 we illustrate the radial velocities difference expected between the interstellar and circumstellar DBs for the HV target IRAS 19114+0002. The narrow $5850 \AA$ CS DB (shifted to the stellar radial velocity) would be completely separated from the observed (IS) DB. The atomic line profiles of sodium (middle panel) and potassium (bottom panel) are shown to indicate the ISM distribution in this line of sight. Note also the narrow sodium emission.

In none of the stars is it possible to assign DBs exclusively to nebular or stellar lines. This strongly supports our conclusion that the DBs detected toward the post-AGB stars in our sample do not originate in their circumstellar envelopes.

When globally considered, the radial velocity analysis here strengthens our proposed scenario, in which the DB carriers are 
not present in the circumstellar envelopes of post-AGB stars, or at least not under the excitation conditions needed to produce the transitions that we identify as DBs in the ISM. Targets that show large velocity differences between interstellar and circumstellar lines and that show significant circumstellar reddening (e.g. IRAS 17086-2403, IRAS 17423-1755, IRAS 18025-3906, and IRAS 18062+2410) provide the best candidates for seeking the presence of (weak) circumstellar DBs separated from the interstellar DBs. Our current spectra are not good enough to search for these weak features next to the observed DIBs. Note that both CS and IS DBs could coexist. And, if separated by more than their FWHM ( 40-60 $\mathrm{km} \mathrm{s}^{-1}$ for narrow DBs), the central velocity of the IS and (possibly) CS DB would not be affected by each other.

\section{Conclusions}

The equivalent widths of 9 DBs commonly found in the ISM have been determined for a representative sample of galactic post-AGB stars displaying a wide variety of observational properties. We present here the results of our extensive survey of DBs in envelopes of evolved stars.

We have carefully disentangled the observed extinction by assessing the expected interstellar extinction for each of the observed targets. This allowed us to select a sub-sample of targets whose line of sight reddenings are dominated $(>50 \%)$ by circumstellar dust. In general, the strengths of the DBs are found to follow the same correlation with $E(B-V)$ as observed in field stars only in those sources showing little circumstellar contribution to the overall reddening. In contrast, DBs are weak or absent in sources dominated by circumstellar reddening, irrespective of the dominant chemistry and spectral type of the central star, although our conclusions should be taken with caution due to the relatively small sample size. The results obtained suggest that the carrier(s) of the DBs does not form, or at least they are not "available" to produce any detectable spectral feature during the post-AGB phase. The carriers, if present in the circumstellar envelope of these stars, are not found under the environmental conditions needed to excite the transitions which we identify as DBs in the ISM. The radial velocity analysis of the features observed in individual sources confirm this result, as the Doppler shifts measured are always found to be consistent with an interstellar origin for the bands observed.

The DB carriers may be carbonaceous species or radicals attached to large organic molecules, trapped in lattice or more complex structures, or constituents of the mantle of circumstellar dust grains that are liberated to the ISM only after strong UV irradiation (either UV photons from the central star or from the more energetic interstellar UV field). In this sense, the identification of the carriers as strongly ionised PAHs and/or radicals liberated from carbonaceous species as a consequence of photoevaporation of dust grains in the ISM looks tempting and would be consistent with our observations. However, we do not find any evidence of the carbonaceous nature of the carrier(s) in our sample stars, something generally accepted in the literature, nor any correlation with the presence of PAHs in the mid-infrared spectrum of these sources, as has been claimed by several authors in the past.

If DBs are connected with PAHs or with any other carbonaceous species such as the ones suggested in the introduction of this paper, their carrier(s) must form at a later stage, probably under different excitation conditions, once the envelope of the post-AGB star is totally diluted in the interstellar medium as a result of the expansion of the shell.
Acknowledgements. Many of the spectra used in the analysis here were kindly provided by Hans van Winckel and Maarten Reyniers, working at the Katholieke Universiteit Leuven, Belgium. The authors are also grateful to Bernard Foing and Nathalie Boudin, who participated in an early stage of this project and with whom we had very fruitful discussions. We sincerely thank the referees for their helpful and constructive comments. This work was partially funded by grants AYA2003-09499 and AYA2004-05382 of the Spanish Ministerio de Ciencia y Tecnología.

\section{References}

Allamandola, L. J., Hudgins, D. M., \& Sandford, S. A. 1999, ApJ, 511, L115 Arellano, A., Giridhar, S., \& Mathias, P. 2001, A\&A, 368, 250

Arkhipova, V. P., Ikonnikova, N., Noskova, R., \& Sokol, G. 2000, AstL, 26, 609 Bakker, E. J., Dishoeck, E. F. V., Waters, L. B. F. M., \& Schoenmaker, T. 1997, A\&A, 323, 469

Bujarrabal, V., Alcolea, J., \& Planesas, P. 1992, A\&A, 257, 701

Clube, K. L., \& Gledhill, T. M. 2004, MNRAS, 355, 17

Cohen, M., \& Jones, B. F. 1987, ApJ, 321, L151

Cox, N. L. J., Kaper, L., Foing, B. H., \& Ehrenfreund, P. 2005, A\&A, 438, 187

Cox, N. L. J., Cordiner, M. A., Ehrenfreund, P., et al. 2007, A\&A, 470, 941

Crawford, M. K., Tielens, A. G. G. M., \& Allamandola, L. J. 1985, ApJ, 293, L45

Desmurs, J., Baudry, A., Sivagnanam, P., \& Henkel, C. 2002, A\&A, 394, 975

Douglas, A. E. 1977, Nature, 269, 130

Drimmel, R., Cabrera-Lavers, A., \& López-Corredoira, M. 2003, A\&A, 409, 205

Ehrenfreund, P., \& Foing, B. H. 1996, A\&A, 307, L25

Ehrenfreund, P., Cami, J., Jiménez-Vicente, J., et al. 2002, ApJ, 576, L117

Fernie, J. D. 1983, ApJS, 52, 7

Fitzgerald, M. P. 1970, A\&A, 4, 234

Foing, B. H., \& Ehrenfreund, P. 1994, Nature, 369, 296

Fujii, F., Nakada, Y., \& Parthasarathy, M. 2002, A\&A, 385, 884

Fulara, J., \& Krelowski, J. 2000, New Astron. Rev., 44, 581

Galazutdinov, G. A., Musaev, F. A., Krelowski, J., \& Walker G. A. H. 2000, PASP, 112, 648

Galazutdinov, G. A., Stachowska, W., Musaev, F. A., et al. 2002, A\&A, 396, 987 García-Lario, P., Manchado, A., Pych, W., \& Pottasch, S. R. 1997a, A\&AS, 126, 479

García-Lario, P., Parthasarathy, M., de Martino, D., et al. 1997b, A\&A, 326, 1103

García-Lario, P., Manchado, A., Ulla, A., \& Manteiga, M. 1999, ApJ, 513, 941

Gauba, G., \& Parthasarathy, M. 2003, A\&A, 407, 1007

Gauba, G., \& Parthasarathy, M. 2004, A\&A, 417, 201

Gauba, G., Parthasarathy, M., Kumar, B., Yadav, R. K. S., \& Sagar, R. 2003, A\&A, 404, 305

Guarinos, J. 1988a, Bull. d'inf. cent. données stellaires, 35, 161

Guarinos, J. 1988b, Bull. d'inf. cent. données stellaires, 34, 141

Guarinos, J. 1997, VizieR On-line Data Catalog: II/135 http://vizier. u-strasbg.fr/viz-bin/VizieR

Heap, S. R., \& Augensen, H. J. 1987, ApJ, 313, 286

Heger, M. L. 1922, Lick Observatory bulletin, 337, 141

Herbig, G. H. 1993, ApJ, 407, 142

Herbig, G. H. 1995, ARA\&A, 33, 19

Hrivnak, B. J. 1995, ApJ, 438, 341

Hrivnak, B. J., \& Kwok, S. 1991, ApJ, 371, 631

Hrivnak, B. J., \& Kwok, S. 1999, ApJ, 513, 869

Hrivnak, B. J., \& Reddy, B. E. 2003, ApJ, 590, 1049

Hrivnak, B. J., Kwok, S., \& Volk, K. 1989, ApJ, 346, 265

Hrivnak, B. J., Kwok, S., \& Su, K. Y. L. 1999, ApJ, 524, 849

Hrivnak, B. J., Volk, K., \& Kwok, S. 2000, ApJ, 535, 275

Hu, J. Y., Slijkhuis, S., de Jong, T., \& Jiang, B. W. 1993a, A\&AS, 100, 413

Hu, J. Y., Slijkhuis, S., Nguyen-Q-Rieu, \& de Jong, T. 1993b, A\&A, 273, 185

Hu, J. Y., te Lintel Hekkert, P., Slijkhuis, F., et al. 1994, A\&AS, 103, 301

Iglesias-Groth, S. 2004, Lecture Notes and Essays in Astrophysics I, 105 Iglesias-Groth, S. 2007, ApJ, 661, L167

Jenniskens, P., \& Désert, F. 1994, A\&AS, 106, 39

Kendall, T. R., Mauron, N., McCombie, J., \& Sarre, P. J. 2002, A\&A, 387, 624 Kerr, T. H., Hibbins, R. E., Fossey, S. J., Miles, J. R., \& Sarre, P. J. 1998, ApJ, 495, 941

Klochkova, V. G., Szczerba, R., Panchuk, V. E., \& Volk, K. 1999, A\&A, 345, 905914

Klochkova, V. G., Szczerba, R., \& Panchuk, V. E. 2000, AstL, 2, 88103

Klochkova, V. G., Yushkin, M. V., Miroshnichenko, A. S., Panchuk, V. E., \& Bjorkman, K. S. 2002, A\&A, 392, 143

Krelowski, J., \& Walker, G. A. H. 1987, ApJ, 312, 860

Krelowski, J., Schmidt, M., \& Snow, T. P. 1997, PASP, 109, 1135

Krelowski, J., Galazutdinov, G. A., \& Musaev, F. A. 1998, ApJ, 493, 217

Kwok, S., Hrivnak, B. J., \& Geballe, T. R. 1995, ApJ, 454, 394 
Kwok, S., Volk, K, \& Hrivnak, B. J. 1999, IAU Symp., 191, 297

Le Bertre, T. 1990, A\&A, 236, 472

Le Bertre, T., \& Lequeux, J. 1992, A\&A, 255, 288

Le Bertre, T., \& Lequeux, J. 1993, A\&A, 274, 909

Leger, A., \& D'Hendecourt, L. 1985, A\&A, 146, 81

Lewis, B. M. 2000, ApJ, 533, 959

Maas, T., van Winckel, H., Evans, T. L., et al. 2003, A\&A, 405, 271

Malfait, K., Bogaert, E., \& Waelkens, C. 1998, A\&A, 331, 211

Mauron, N., \& Kendall, T. R. 2004, A\&A, 428, 535

McCall, B. J., Oka, T., Thorburn, J., Hobbs, L., \& York, D. G. 2002, ApJ, 567, 145

Megier, A., Krelowski, J., \& Weselak, T. 2005, MNRAS, 358, 563

Meixner, M., Ueta, T., Dayal, A., et al. 1999, ApJS, 122, 221

Merrill, P. W. 1936, ApJ, 83, 126

Neckel, T., Klare, G., \& Sarcander, M. 1995, VizieR Online Data Catalog, 2062 Oudmaijer, R. D., van der Veen, W. E. C. J., Waters, L. B. F. M., et al. 1992, A\&AS, 96, 625

Parthasarathy, M., García-Lario, P., Sivarania, T., Manchado, A., \& Sanz Fernández de Córdoba, L. 2000a, A\&A, 357, 241

Parthasarathy, M., Vijapurkar, J., \& Drilling, J. S. 2000b, A\&AS, 145, 269

Pottasch, S. R., Bernard-Salas, J., Beintema, D. A., \& Feibelman, W. A. 2004, A\&A, 423, 593

Prichet, C. J., \& Grillmair, C. J. 1984, PASP, 96, 349

Reddy, B. E., \& Parthasarathy, M. 1996, AJ, 112, 2053

Reddy, B. E., Bakker, E. J., \& Hrivnak, B. J. 1999, ApJ, 524, 831

Reed, B. C., \& Vance, S. J. 1996, AJ, 112, 2855

Reyniers, M., van Winckel, H., Gallino, R., \& Straniero, O. 2004, A\&A, 417, 269

Ruiterkamp, R., Cox, N. L. J., Spaans, M., et al. 2005, A\&A, 432, 515

Salama, F., Bakes, E. L. O., Allamandola, L. J., \& Tielens, A. G. G. M. 1996, ApJ, 458, 621
Salama, F., Galazutdinov, G. A., Krelowski, J., et al. 1999, ApJ, 526, 265

Sarre, P. J. 2006, J. Mol. Spec., 238, 1

Sarre, P. J., Miles, J. R., Kerr, T. H., et al. 1995, MNRAS, 277, L41

Scarrott, S. M., Watkin, S., Miles, J. R., \& Sarre, P. J. 1992, MNRAS short communication, 255, 11

Snow, Jr., T. P. 1973, PASP, 85, 590

Snow, Jr., T. P., \& Wallerstein, G. 1972, PASP, 84, 492

Suárez, O. 2004, Ph.D. Thesis, Universidade de Vigo, Spain

Suárez, O., García-Lario, P., Manchado, A., et al. 2006, A\&A, 458, 173

te Lintel Hekkert, P., Caswell, J. L., Habing, H. J., Haynes, R. F., \& Norris, R. P. 1991, A\&AS, 90, 327

Thorburn, J. A., Hobbs, L. M., McCall, B. J., et al. 2003, ApJ, 584, 339356

Torres-Peimbert, S., Peimbert, M., \& Daltabuit, E. 1980, ApJ, 238, 133

Turner, D. G., \& Drilling, J. S. 1984, PASP, 96, 292

Van de Steene, G. C. V., \& van Hoof, P. 2003, A\&A, 406, 773

Van der Veen, W. E. C. J., Habing, H. J., \& Geballe, T. R. 1989, A\&A, 226, 108

Van der Zwet, G. P., \& Allamandola, L. J. 1985, A\&A, 146, 76

Van Winckel, H. 1997, A\&A, 319, 561

Van Winckel, H., \& Reyniers, M. 2000, A\&A, 354, 135

Volk, K., \& Kwok, S. 1989, ApJ, 342, 345

Waters, L. B. F. M., Lamers, H. J. G. L. M., Snow, T. P., et al 1989, A\&A, 211, 208

Weselak, T., Fulara, J., Schmidt, M. R., \& Krelowski, J. 2001, A\&A, 377, 677

Woodsworth, A. W., Kwok, S., \& Chan, S. J. 1990, 228, 503

Začs, L., Schmidt, M. R., \& Szczerba, R. 1999a, MNRAS, 306, 903

Začs, L., Schmidt, M. R., Szczerba, R., \& Spēlmanis, R. 1999b, ASPC, 188, 221

Začs, L., Spēlmanis, R., Schmidth, M. R., \& Szczerba, R. 2001, ASP Conf. Ser., 223,1645

Začs, L., Spēlmanis, R., Musaev, F. A., \& Galazutdinov, G. A. 2003, MNRAS, 339,460 
R. Luna et al.: A search for diffuse bands in post-AGB stars, Online Material p 1

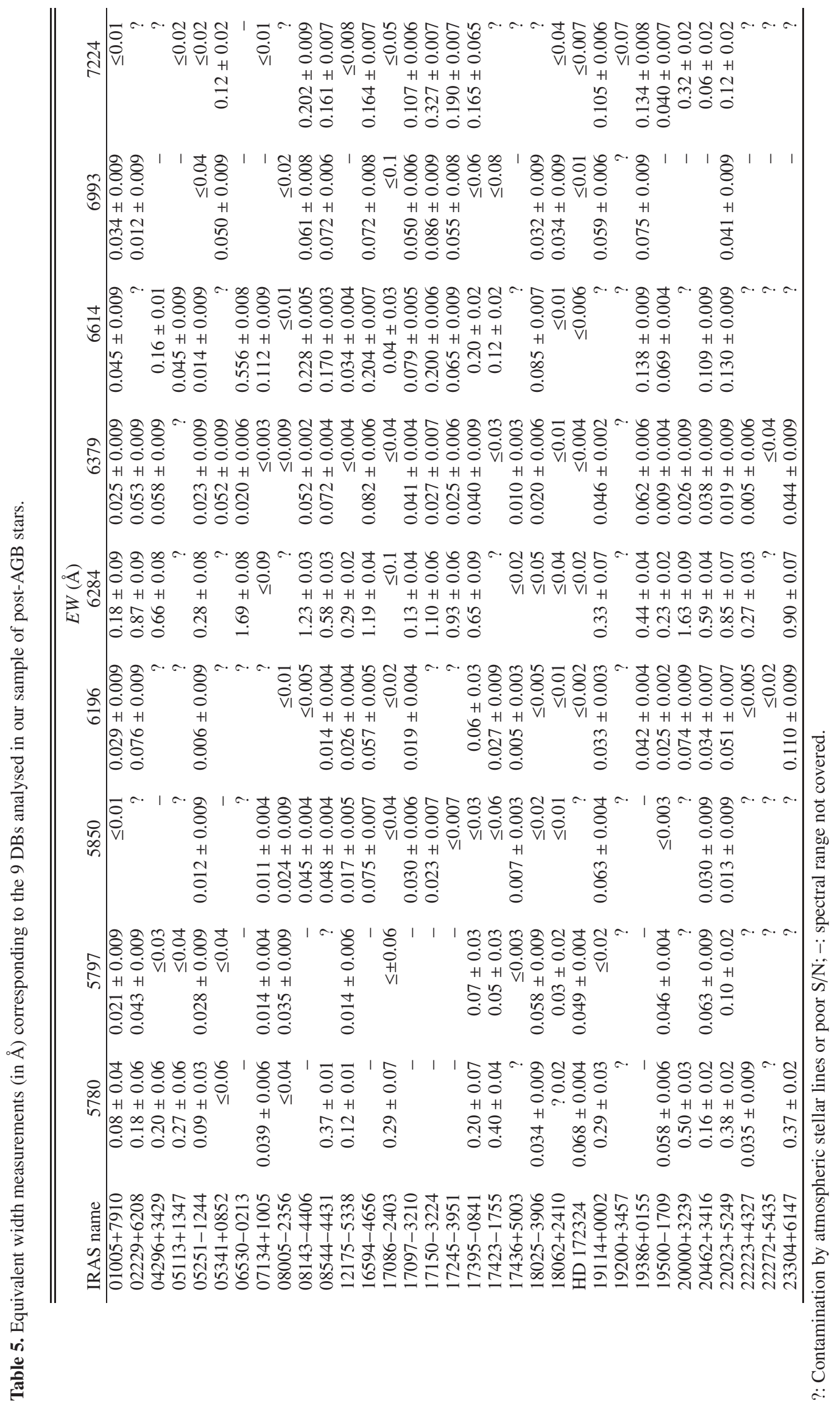


R. Luna et al.: A search for diffuse bands in post-AGB stars, Online Material $p 2$

Table 6. Radial velocity measurements (in $\mathrm{km} \mathrm{s}^{-1}$ ) associated to several atmospheric stellar and nebular lines detected in the post-AGB stars of our sample.

\begin{tabular}{|c|c|c|c|c|c|c|c|c|c|c|c|}
\hline IRAS Name & $\begin{array}{l}\mathrm{H} \alpha \\
6563\end{array}$ & $\begin{array}{c}\mathrm{He} \mathrm{I} \\
6678\end{array}$ & $\overline{\mathrm{C}}$ & $\overline{\mathrm{N}}$ & $\mathrm{O}$ & $\overline{\mathrm{Si}}$ & $\overline{\mathrm{Fe}}$ & $\begin{array}{l}{[\mathrm{N} \text { II] }} \\
6548\end{array}$ & $\begin{array}{l}\mathrm{N} \text { II] } \\
6583\end{array}$ & $\begin{array}{l}\text { S II }] \\
6716\end{array}$ & $\begin{array}{l}\overline{[\mathrm{S}} \text { II }] \\
6730\end{array}$ \\
\hline $01005+7910$ & $-34 \mathrm{e}$ & $-76 \mathrm{P}$ & -31 & -37 & -32 & -38 & - & - & - & -41 & -38 \\
\hline $02229+6208$ & $29 \mathrm{a}$ & - & - & - & 23 & 21 & 26 & - & - & - & - \\
\hline $04296+3429$ & $4 \mathrm{a}$ & $-11 \mathrm{a}$ & - & -4 & -8 & -4 & -9 & - & - & - & - \\
\hline $05113+1347$ & $-4 \mathrm{a}$ & - & -7 & -13 & -13 & -14 & -10 & - & - & - & - \\
\hline $05251-1244$ & $44 \mathrm{e}$ & $40 \mathrm{e}$ & 41 & - & - & 44 & - & 32,57 & 31,55 & 31,57 & 32,58 \\
\hline $05341+0852$ & 39 a & 18 a & 44 & 22 & 20 & 29 & 23 & - & - & - & _- \\
\hline $06530-0213$ & 39 a & $22 \mathrm{a}$ & 36 & 30 & 34 & 33 & 39 & - & - & - & - \\
\hline $07134+1005$ & $54 \mathrm{a}$ & - & 68 & 87 & 90 & 84 & 67 & - & - & - & - \\
\hline 08005-2356 & $85 \mathrm{P}$ & $59 \mathrm{P}$ & 34 & 27 & 52 & 35 & 35 & - & - & - & - \\
\hline 08143-4406 & $40 \mathrm{a}$ & - & 66 & 40 & 38 & 35 & 37 & - & - & - & - \\
\hline 08544-4431 & $90 \mathrm{P}$ & $43 \mathrm{a}$ & 45 & 45 & 54 & 46 & 48 & - & - & - & - \\
\hline $12175-5338$ & $28 \mathrm{a}$ & - & - & - & - & - & - & - & - & - & - \\
\hline $16594-4656$ & $15 \mathrm{P}$ & $-2 \mathrm{a}$ & -14 & -14 & -22 & -19 & -17 & - & - & -7 & -7 \\
\hline 17086-2403 & $99 \mathrm{e}$ & - & 120 & 93 & 97 & 99 & 107 & 95 & 94 & 97 & 96 \\
\hline $17097-3210$ & $35 \mathrm{a}$ & $15 \mathrm{a}$ & 26 & 39 & -18 & - & -1 & - & - & - & - \\
\hline $17150-3224^{*}$ & $5 \mathrm{P}$ & - & 41 & 52 & 32 & 40 & 3 & 8 & 5 & 6 & 6 \\
\hline $17245-3951^{*}$ & $14 \mathrm{P}$ & $-83 \mathrm{a}$ & -36 & -92 & -81 & -76 & -77 & 20 & 20 & -16 & -14 \\
\hline 17395-0841 & $87 \mathrm{e}$ & $87 \mathrm{e}$ & - & - & - & - & 66 & 113 & 113 & - & - \\
\hline $17423-1755$ & $96 \mathrm{e}$ & $83 \mathrm{e}$ & 76 & 73 & 83 & 79 & - & - & - & - & - \\
\hline $17436+5003$ & $-55 \mathrm{a}$ & $-56 \mathrm{a}$ & -48 & -50 & -48 & -48 & -50 & - & - & - & - \\
\hline 18025-3906 & $-104 \mathrm{a}$ & $-89 a$ & -88 & -111 & -80 & -94 & -101 & - & - & - & - \\
\hline $18062+2410$ & $78 \mathrm{e}$ & $30 \mathrm{a}$ & 88 & 72 & 74 & 70 & - & 70 & 69 & 72 & 70 \\
\hline HD 172324 & $-72 \mathrm{e}$ & $-117 \mathrm{a}$ & -67 & -108 & -112 & -115 & -115 & - & - & - & - \\
\hline $19114+0002$ & $110 \mathrm{a}$ & 89 a & 125 & 114 & 106 & 101 & 102 & - & - & - & - \\
\hline $19200+3457$ & $7 \mathrm{P}$ & $-5 \mathrm{a}$ & -8 & 5 & 1 & 3 & - & - & - & - & - \\
\hline $19386+0155$ & $25 \mathrm{a}$ & $15 \mathrm{a}$ & 19 & 25 & 29 & 25 & 24 & - & - & - & - \\
\hline 19500-1709 & $20 \mathrm{P}$ & $4 \mathrm{a}$ & - & 24 & 24 & 27 & 25 & - & - & - & - \\
\hline $20000+3239$ & $22 \mathrm{a}$ & $6 \mathrm{a}$ & 18 & 13 & 11 & 4 & 11 & - & - & - & - \\
\hline $20462+3416$ & $-68 \mathrm{e}$ & $-44 \mathrm{P}$ & -76 & -79 & -92 & -75 & - & -73 & -77 & -75 & -76 \\
\hline $22023+5249$ & $-136 \mathrm{e}$ & $-114 \mathrm{e}$ & -122 & -139 & -133 & -133 & -131 & -134 & -136 & -133 & -134 \\
\hline $22223+4327$ & $-24 \mathrm{e}$ & $-37 \mathrm{a}$ & -12 & -27 & -25 & -28 & -28 & - & - & - & - \\
\hline $22272+5435$ & $-28 \mathrm{a}$ & $-38 a$ & -35 & - & -32 & -37 & -26 & - & - & - & - \\
\hline $23304+6147$ & $-15 \mathrm{a}$ & - & - & -16 & -15 & - & - & - & - & - & - \\
\hline
\end{tabular}

P: P-Cygni profile; a: absorption; e: emission; *: different atmospheric and nebular velocities. 
R. Luna et al.: A search for diffuse bands in post-AGB stars, Online Material $p 3$

Table 7. Radial velocity measurements (in $\mathrm{km} \mathrm{s}^{-1}$ ) associated to the Na ID (5889.95 and $5895.92 \AA$ ) and K I (7698.97 $\AA$ ) lines detected in the post-AGB stars of our sample.

\begin{tabular}{|c|c|c|c|c|c|c|}
\hline \multirow[b]{2}{*}{ IRAS name } & \multicolumn{2}{|c|}{ KI $7699 \AA$} & \multicolumn{2}{|c|}{ Na I $5890 \AA ̊$} & \multicolumn{2}{|c|}{ Na I $5896 \AA ̊$} \\
\hline & emission & absorption & emission & absorption & emission & absorption \\
\hline $01005+7910^{*}$ & n.d. & $1,-6$ & 20 & $-65,-41,-1$ & 21 & $-65,-42,-1$ \\
\hline $02229+6208$ & n.d. & 12,29 & n.d. & $-2,25$ & n.d. & $-2,24$ \\
\hline $04296+3429$ * & -7 & $-18,58$ & 21 & $-17,6,56$ & 21 & $-16,6,56$ \\
\hline $05113+1347 *$ & n.d. & $-17,2$ & -44 & $-17,6$ & -43 & $-18,5$ \\
\hline $05251-1244$ & n.d. & 6 & n.d. & $4,-11$ & n.d. & $5,-11$ \\
\hline $05341+0852$ & n.d. & 14 & 23 & 21 & 23 & 21 \\
\hline $06530-0213$ & - & - & n.d. & 25,47 & n.d. & 25,46 \\
\hline $07134+1005$ & n.d. & 93 & n.d. & $29,39,91$ & n.d. & $28,40,92$ \\
\hline $08005-2356$ * & n.d. & $24,-8,48$ & -30 & $-47,19$ & -30 & $-52,17$ \\
\hline $08143-4406$ & n.d. & $19,4,35$ & n.d. & $16,42,61$ & n.d. & $17,42,61$ \\
\hline 08544-4431 & n.d. & 27 & n.d. & $-42,15,39$ & n.d. & $-41,16,43$ \\
\hline $12175-5338$ & n.d. & -5 & 31 & $-27,-6,31$ & 32 & $-27,-5,32$ \\
\hline $16594-4656^{*}$ & n.d. & $-10,-41,5$ & 14 & $-29,6$ & 13 & $-29,8$ \\
\hline $17086-2403^{*}$ & n.d. & 12 & -6 & 17 & -5 & 14 \\
\hline $17097-3210$ & n.d. & 5 & n.d. & $-29,3$ & n.d. & $-29,3$ \\
\hline $17150-3224$ & $10,21,2$ & n.d. & 10 & 5 & 10 & 2 \\
\hline $17245-3951$ & $-1,27$ & $-86,-57$ & 19 & $-98,-63,-31$ & 19 & $-98,-66,-31$ \\
\hline $17395-0841$ & n.d. & 17,3 & 2 & -1 & 3 & -2 \\
\hline $17423-1755$ & n.d. & 11 & 3,72 & n.d. & 3,71 & n.d. \\
\hline $17436+5003$ & n.d. & -50 & n.d. & $-66,-19$ & n.d. & $-65,-19$ \\
\hline 18025-3906 & n.d. & $-89,-73$ & n.d. & $-121,-34,8$ & n.d. & $-121,-31,10$ \\
\hline $18062+2410$ & - & - & 75 & 3,20 & 67 & 3,13 \\
\hline HD 172324 & n.d. & 6 & n.d. & $-28,7$ & n.d. & $-28,8$ \\
\hline 19114+0002 & n.d. & $10,67,83$ & n.d. & $11,64,127$ & n.d. & $12,65,123$ \\
\hline $19200+3457$ & - & - & 6 & 8 & 7 & 9 \\
\hline $19386+0155$ & n.d. & 10 & - & - & - & - \\
\hline 19500-1709 & n.d. & $4,22,-9$ & n.d. & $2,12,20$ & n.d. & $2,14,22$ \\
\hline $20000+3239$ & n.d. & 2,13 & n.d. & $-2,21$ & n.d. & 2,21 \\
\hline $20462+3416$ & n.d. & $-21,-8$ & n.d. & $-25,-6,19$ & n.d. & $-26,-7,17$ \\
\hline $22023+5249$ & n.d. & -23 & $27,-130$ & $-46,2$ & $27,-131$ & $-46,4$ \\
\hline $22223+4327$ & n.d. & $-2,-30,-43$ & n.d. & $-42,-18,0$ & n.d. & $-44,-18,0$ \\
\hline $22272+5435$ & n.d. & -41 & n.d. & $-41,-4$ & n.d. & $-41,-4$ \\
\hline $23304+6147^{*}$ & n.d. & $-1,-12,-29$ & 24 & $-52,-29,-7$ & 24 & $-53,-29,-7$ \\
\hline
\end{tabular}

*: Emission over absorption; -: spectral range not covered; n.d.: non detected. 
R. Luna et al.: A search for diffuse bands in post-AGB stars, Online Material p 4

Table 8. Radial velocity measurements (in $\mathrm{km} \mathrm{s}^{-1}$ ) associated to the DBs detected in the post-AGB stars of our sample.

\begin{tabular}{|c|c|c|c|c|c|c|c|c|c|}
\hline \multirow[b]{2}{*}{ IRAS name } & \multicolumn{9}{|c|}{ Diffuse band } \\
\hline & 5780 & 5797 & 5850 & 6196 & 6284 & 6379 & 6614 & 6993 & 7224 \\
\hline $01005+7910$ & -16 & $29^{*}$ & - & -2 & -9 & 2 & -12 & -16 & - \\
\hline $02229+6208$ & 32 & $-3^{*}$ & - & 33 & 52 & 22 & - & 24 & - \\
\hline $04296+3429$ & 66 & - & - & - & 55 & 59 & 60 & - & - \\
\hline $05113+1347$ & 8 & - & - & - & - & - & 0 & - & - \\
\hline $05251-1244$ & -3 & 11 & 20 & 0 & 29 & -14 & 0 & - & - \\
\hline $05341+0852$ & - & - & - & - & - & 4 & - & 15 & 37 \\
\hline $06530-0213$ & - & - & - & - & 26 & 33 & 12 & - & - \\
\hline $07134+1005$ & -5 & -14 & 2 & - & - & - & 5 & - & - \\
\hline $08005-2356$ & - & 30 & 36 & - & - & - & - & - & - \\
\hline 08143-4406 & - & - & 6 & - & 20 & 12 & 24 & 16 & 16 \\
\hline 08544-4431 & 23 & - & 16 & $-45^{*}$ & 19 & 17 & 28 & 5 & 8 \\
\hline $12175-5338$ & 11 & 2 & -8 & -7 & -6 & - & -4 & - & - \\
\hline $16594-4656$ & - & - & 9 & 6 & 9 & 6 & 2 & -4 & -3 \\
\hline $17086-2403$ & 14 & - & - & - & - & - & 2 & - & - \\
\hline $17097-3210$ & - & - & 3 & 5 & 5 & -4 & 5 & 13 & 0 \\
\hline $17150-3224$ & - & - & -1 & - & -2 & 0 & 9 & -3 & 1 \\
\hline $17245-3951$ & - & - & - & - & 27 & -7 & 5 & 16 & 22 \\
\hline $17395-0841$ & -10 & 17 & - & 8 & 0 & 3 & 3 & - & -4 \\
\hline $17423-1755$ & 15 & 9 & - & 9 & - & - & 12 & - & - \\
\hline $17436+5003$ & - & - & 23 & 0 & - & 19 & - & - & - \\
\hline $18025-3906$ & 7 & $-35^{*}$ & - & - & - & $-36^{*}$ & 24 & 19 & - \\
\hline $18062+2410$ & - & 15 & - & - & - & - & - & -1 & - \\
\hline HD 172324 & 39 & $-29^{*}$ & - & - & - & - & - & - & - \\
\hline $19114+0002$ & 34 & - & 6 & 19 & 12 & 8 & - & -5 & 29 \\
\hline $19200+3457$ & - & - & - & - & - & - & - & - & - \\
\hline $19386+0155$ & - & - & - & 5 & 8 & 3 & 6 & 4 & 9 \\
\hline 19500-1709 & 11 & $-44^{*}$ & - & 4 & 6 & 3 & $35^{*}$ & - & 9 \\
\hline $20000+3239$ & 15 & - & - & 6 & 35 & 1 & - & - & 12 \\
\hline $20462+3416$ & -14 & -4 & $45^{*}$ & -14 & 5 & -13 & -9 & - & -2 \\
\hline $22023+5249$ & -21 & -7 & $37^{*}$ & -14 & -17 & 2 & 2 & 0 & -2 \\
\hline $22223+4327$ & 17 & - & - & - & 6 & 2 & - & - & - \\
\hline $22272+5435$ & 39 & - & - & - & - & - & - & - & - \\
\hline $23304+6147$ & -3 & - & - & -26 & 5 & -8 & - & - & - \\
\hline
\end{tabular}

*: Likely contaminated measurement. 